\author{
Aus der Abteilung Anaesthesiologie I \\ (Ehem. Leiter: Prof. Dr. med. B.M. Graf) \\ im Zentrum Anaesthesiologie, Rettungs- und Intensivmedizin \\ der Medizinischen Fakultät der Universität Göttingen
}

\title{
Einfluss der Anästhesie auf das postoperative Auftreten des Restless Legs Syndroms
}

\author{
INAUGURAL- DISSERTATION \\ zur Erlangung des Doktorgrades \\ der Medizinischen Fakultät der Georg-August-Universität zu Göttingen
}

vorgelegt von

Désirée Karimdadian

aus Teheran/ Iran

Göttingen 2009 


\section{Inhaltsverzeichnis}

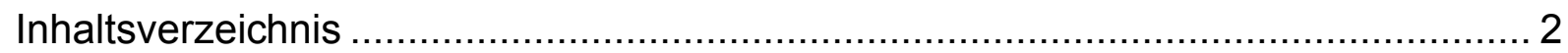

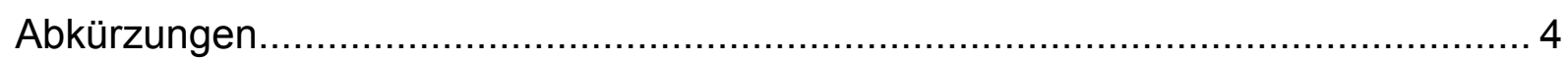

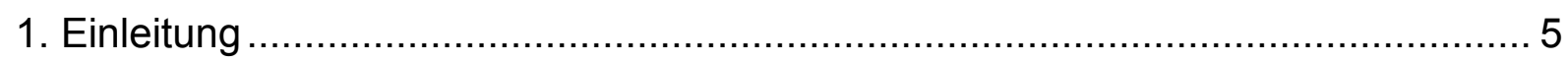

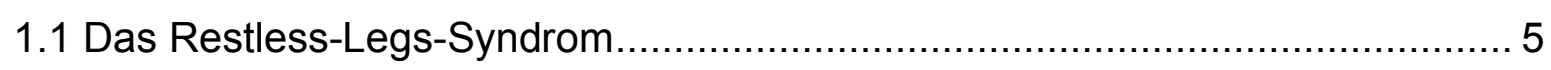

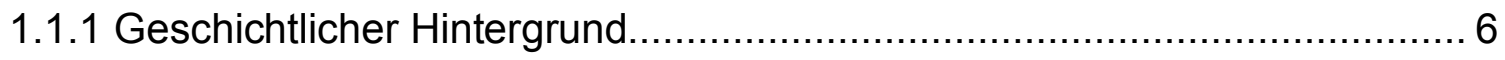

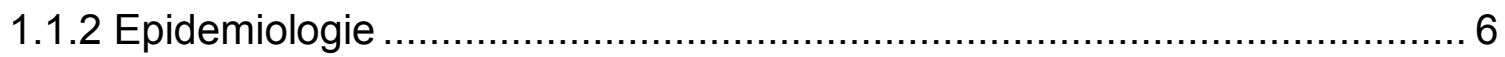

1.1.3 Klassifikation und Erblichkeit .......................................................... 7

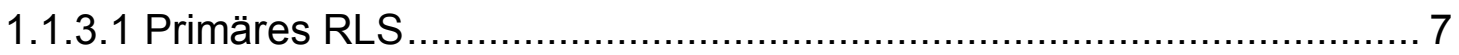

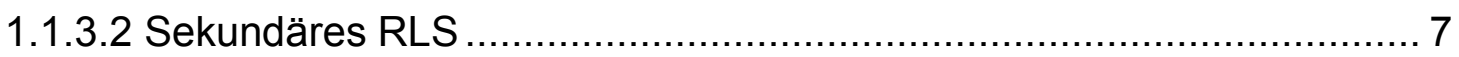

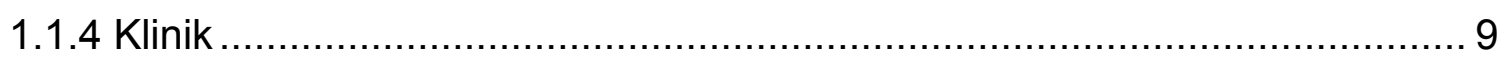

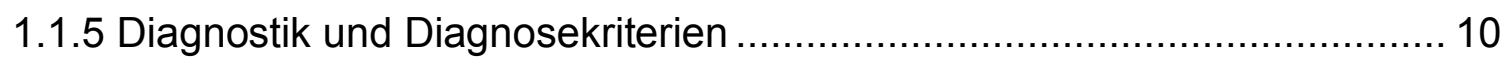

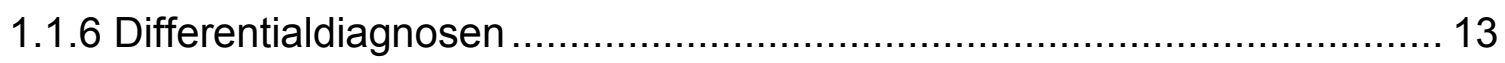

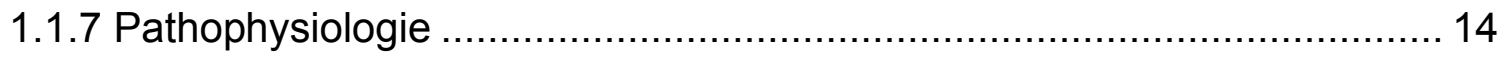

1.1.8 Therapie

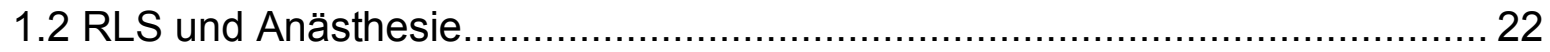

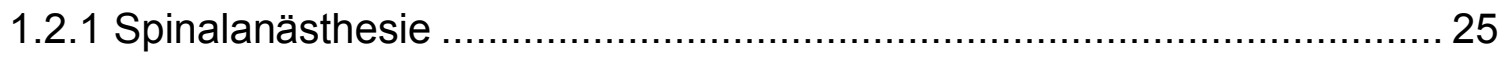

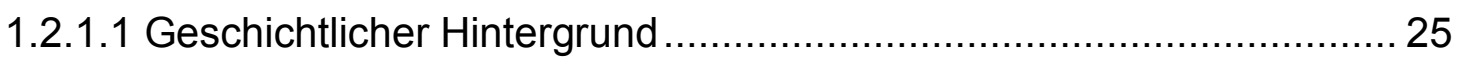

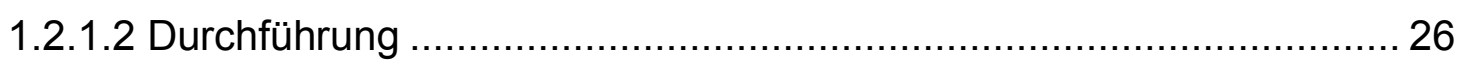

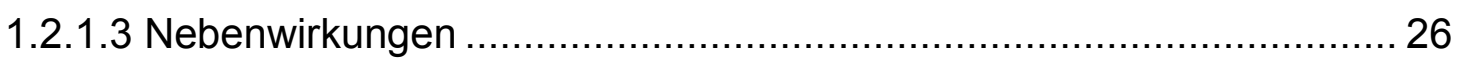

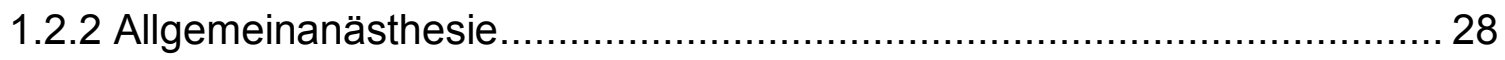

1.2.3 Lokalanästhesie und periphere Regionalanästhesie .............................. 28

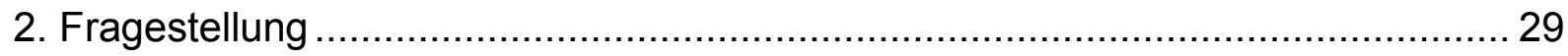

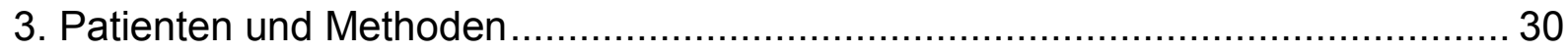

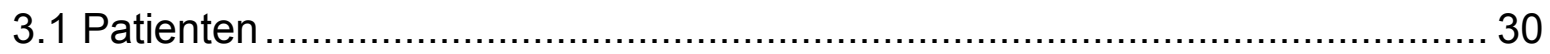




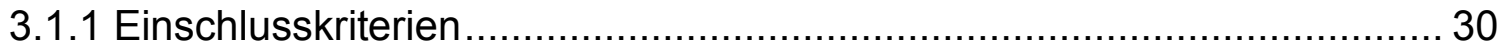

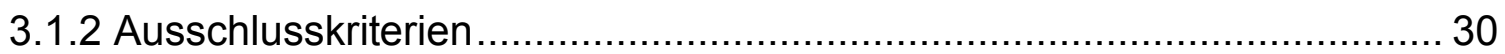

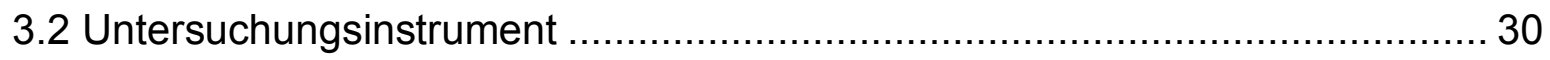

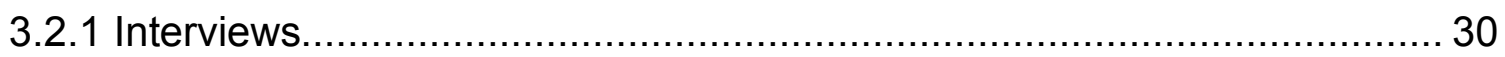

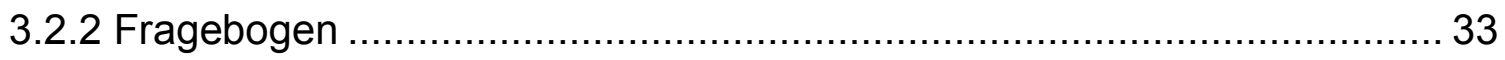

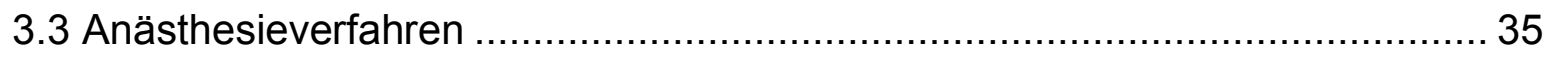

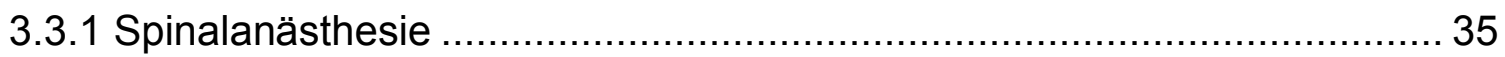

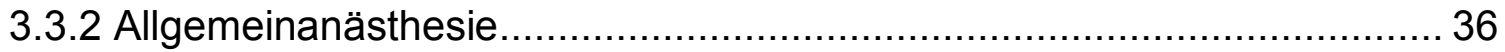

3.3.3 Lokalanästhesie und periphere Regionalanästhesie ............................. 36

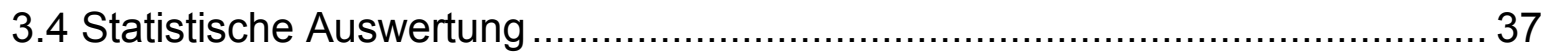

4. Ergebnisse

4.1 Demographische Daten des Patientenkollektivs ........................................ 38

4.1.1 Gruppe der Patienten mit Spinalanästhesie........................................ 39

4.1.2 Gruppe der Patienten mit Allgemeinanästhesie.................................... 40

4.1.3 Gruppe der Patienten mit Lokalanästhesie oder peripherer

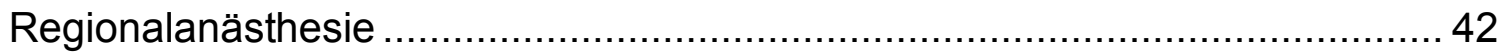

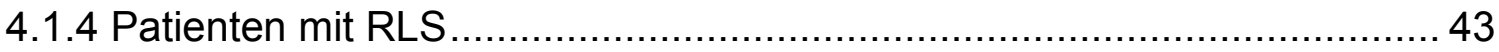

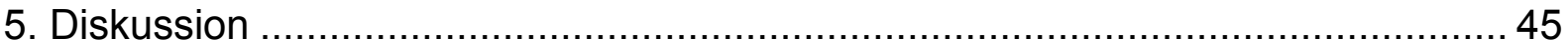

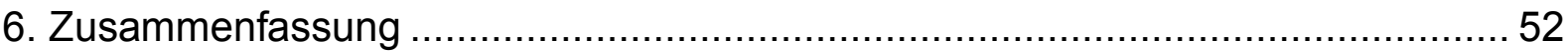

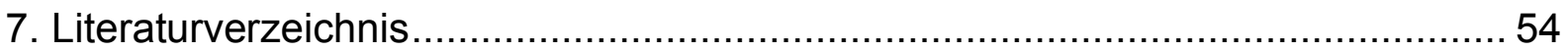

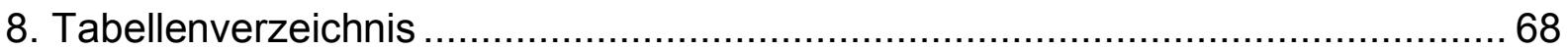

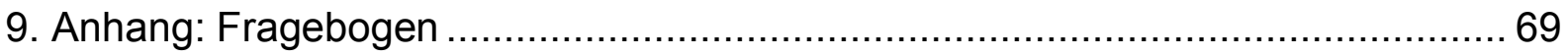




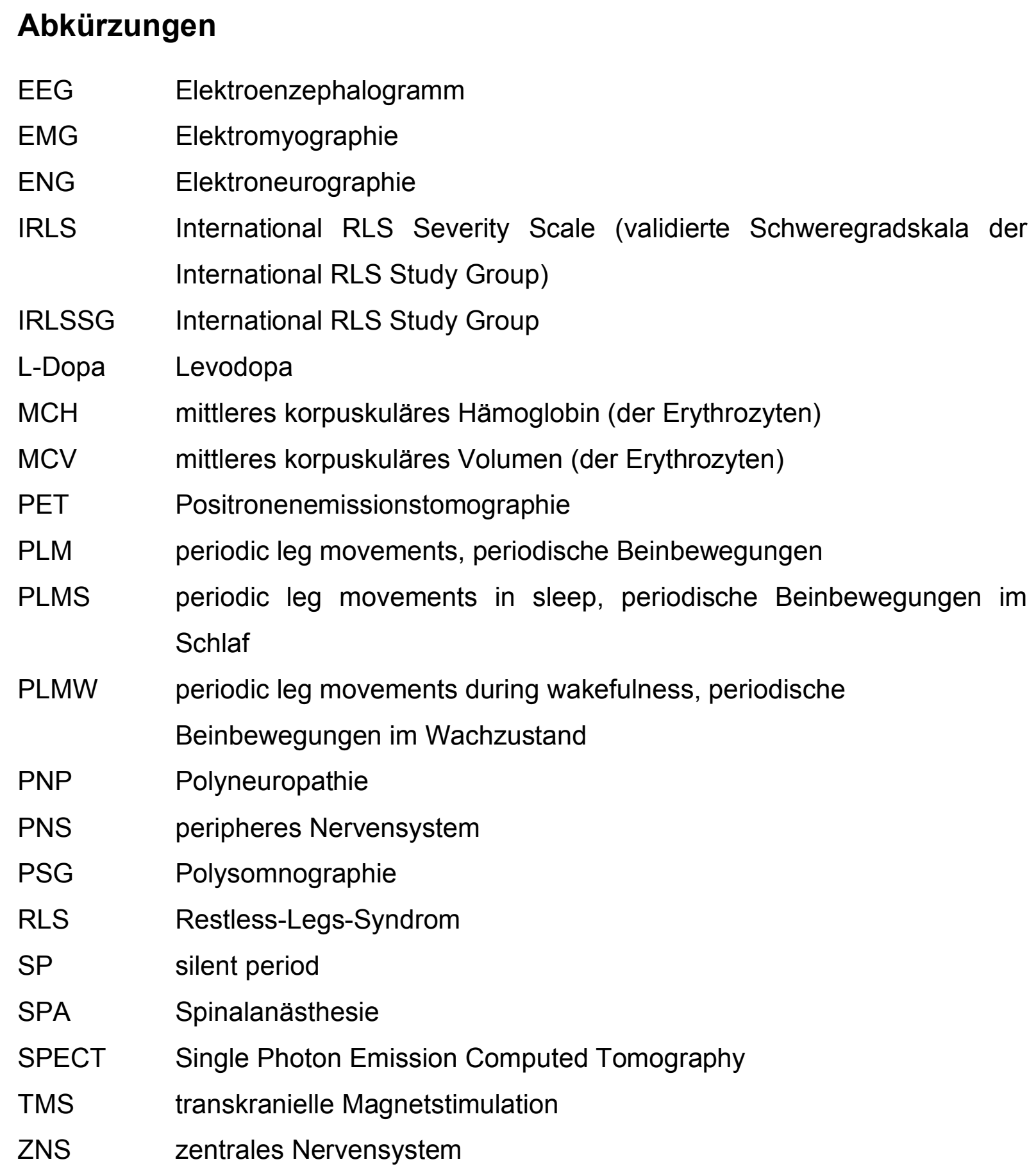




\section{Einleitung}

\subsection{Das Restless-Legs-Syndrom}

Das Restless-Legs-Syndrom (RLS oder Syndrom der ruhelosen Beine) ist eine häufige chronische neurologische Erkrankung mit einer sensorischen und einer motorischen Komponente. Die Patienten klagen über Parästhesien und einem Bewegungsdrang, besonders der Beine. Bei Bewegung kommt es zu einer Linderung der Symptome. Typisch sind periodische Beinbewegungen im Schlaf (periodic leg movements in sleep oder PLMS) oder solche während des Tages (periodic leg movements during awakefulness oder PLMW).

Die Prävalenz schwankt je nach Ethnie zwischen 3 und 10\% und nimmt mit dem Alter zu (Berger et al. 2004). Die Ätiologie ist noch weitgehend unbekannt. Man geht von einer Beteiligung des dopaminergen und opioidergen Neurotransmittersystems aus und unterscheidet zwischen einer primären (ohne fassbare Ursache) und einer sekundären Form des RLS (Allen, Earley 2001; Paulus et al. 2007). Bei der sekundären Form sind die auslösenden Faktoren bekannt.

Seit nun mehr über 300 Jahren wird nach den Ursachen, der Pathophysiologie und einer kausalen Therapie des RLS geforscht. Von einem endgültigen Ergebnis ist man allerdings noch entfernt.

Diese Ungewissheit schlägt sich sowohl in der Forschung als auch in Empfehlungen an RLS-Patienten nieder. Wie weiter unten näher ausgeführt, wird eine Reihe von anästhesierelevanten Pharmaka angeschuldigt, ein RLS auszulösen oder zu aggravieren, ohne dass diesbezüglich Daten vorliegen. So haben auch Högl et al. (2002) den Zusammenhang zwischen Spinalanästhesie (SPA) und RLS postuliert, den zu überprüfen die vorliegende Studie durchgeführt wurde. 


\subsubsection{Geschichtlicher Hintergrund}

Eine erste detaillierte Beschreibung und Definition des Krankheitsbildes mit eigens durchgeführten klinischen Studien gab der schwedische Neurologe Karl-Axel Ekbom (Ekbom 1945). Er prägte auch den Begriff des RLS. Ihm zu Ehren wurde die Erkrankung auch Ekbom-Syndrom genannt.

Mit großer Wahrscheinlichkeit wurde die Erkrankung aber schon viel früher, nämlich im Jahre 1685, durch den englischen Arzt Sir Thomas Willis das erste Mal beschrieben. Er beobachtete bei seinen Patienten mit Schlafstörungen unruhige Beine und Arme und konnte eine Besserung der Symptome unter Opiumtherapie feststellen (Willis 1685).

In Deutschland sprach man im 19. Jahrhundert bei dieser Art von Symptomen von der „Anxietas tibiarum“ (lat. Ängstlichkeit der Schienbeine) und brachte sie mit der Hysterie in Verbindung. Erste Dokumentationen eines RLS-ähnlichen Syndroms nahm der deutsche Neurologe Wittmaack 1861 in seinem Lehrbuch der Nervenkrankheiten vor (Wittmaack 1861).

\subsubsection{Epidemiologie}

Das RLS als neurologisches Krankheitsbild findet sich bei 3 bis $10 \%$ der Gesamtbevölkerung, je nach untersuchter Population und Ethnie (Ekbom 1945 und 1960; Lavigne und Montplaisir 1994; Phillips et al. 2000; Rothdach et al. 2000). Bei Koreanern und Indern ist das RLS mit einer Prävalenz von 3,9\% bzw. 2,1\% eher selten (Cho et al. 2008; Rangarajan et al. 2007), wohingegen es z.B. in Deutschland bei 10,6\% der Bevölkerung auftritt (Berger et al. 2004). Auch die nicht einheitlich verwendeten Diagnosekriterien und unterschiedlichen Methoden tragen zu den stark schwankenden Häufigkeitsangaben bei.

Man beobachtet eine steigende Prävalenz mit zunehmendem Alter, wobei Frauen generell häufiger betroffen sind als Männer (Nichols et al. 2003; Rothdach et al. 
2000; Zucconi und Ferini-Strambi 2004), in der Studie von Berger et al. (2004) sogar doppelt so häufig, wobei das Risiko mit der Anzahl der Geburten ansteigt.

\subsubsection{Klassifikation und Erblichkeit}

Klassifiziert wird das RLS in eine primär oder idiopathisch und in eine sekundär auftretende Erkrankung, wobei es keinen Unterschied im Erscheinungsbild gibt.

\subsubsection{Primäres RLS}

Das primäre RLS kann familiär gehäuft oder sporadisch auftreten. Eine positive Familienanamnese findet man je nach Stichprobe bei 40 bis $80 \%$ der RLS-Patienten (Lavigne und Montplaisir 1994; Walters et al. 1996; Winkelmann et al. 2000). Die primäre familiäre Form manifestiert sich meist schon vor dem 30.Lebensjahr, also deutlich früher als die sporadische Form (Bassetti et al. 2001; Winkelmann et al. 2000). Die Genetik des RLS wird weiter unten im Detail beschrieben (siehe 1.1.7).

In 10 bis $20 \%$ der Fälle treten die Symptome des RLS bereits im Kindesalter auf und werden oft als „Hyperaktivitätssyndrom“ oder „Wachstumsschub“ fehlgedeutet (Picchietti DL und Walters 1996). Auch bei Kindern und Jugendlichen sind die Symptome mit periodischen Beinbewegungen (periodic leg movements=PLM) assoziiert (Picchietti MA und Picchietti DL 2008).

\subsubsection{Sekundäres RLS}

Beim sekundären RLS sind verschiedene internistische und neurologische Erkrankungen, eine Schwangerschaft, aber auch eine Reihe von Medikamenten für die Auslösung der typischen Symptome verantwortlich. Meist sind diese vorübergehend und können durch eine geeignete Therapie gelindert oder sogar völlig behoben werden. 
Zu den häufigsten sekundären Formen gehört das RLS bei Urämie. Man beobachtet es bei bis zu $45 \%$ der dialysepflichtigen niereninsuffizienten Patienten (ColladoSeidel et al. 1998; Siddiqui et al. 2005), wobei die Prävalenz nach Nierentransplantation abnimmt (Kavanagh et al. 2004; Molnar et al. 2005).

Weiterhin findet man eine erhöhte Prävalenz des sekundären RLS bei Eisenmangelanämie (Sun et al. 1998). In einigen Studien konnte gezeigt werden, dass es unter Eisensubstitution zu einer Besserung der Symptome kommt (Earley et al. 2005; O'Keeffe et al. 1994); in anderen Studien konnte dies nicht bestätigt werden (Siddiqui et al. 2005). Andere Autoren gehen von einer Dysfunktion der Regulierungsmechanismen im Eisenstoffwechsel und einem gestörten Transport ins ZNS aus (Berger et al. 2002), da bei Patienten, im Gegensatz zum Serum, erniedrigte Ferritin- und Eisenkonzentrationen im Liquor und speziell in der Substantia nigra gefunden wurden (Mizuno et al. 2005).

In der Schwangerschaft leiden über 20\% aller Frauen an den Symptomen dieser Erkrankung (Goodman et al. 1988), wobei eine Zunahme im dritten Trimenon und ein völliges Verschwinden nach der Entbindung beschrieben wird (Manconi et al. 2004). Als Ursachen wurden ein erniedrigter Eisen-, Folsäure- und Hämoglobinspiegel identifiziert (Tunç et al. 2007).

Ein vermehrtes Auftreten des sekundären RLS beobachteten Salih et al. (1994) bei der rheumatoiden Arthritis. Im Gegensatz dazu sahen Ondo et al. (2000) keinen kausalen Zusammenhang zwischen rheumatologischen Erkrankungen und dem RLS.

In verschiedenen Studien wurde außerdem eine Häufung des RLS bei neurologischen Erkrankungen gefunden. Hierzu gehören zum Beispiel die Polyneuropathien (PNP) (Gemignani und Brindani 2007; Gemignani und Marbini 2002) - besonders betroffen sind hier Diabetiker (Merlino et al. 2007) - die Multiple Sklerose (Auger et al. 2005) und der Morbus Parkinson (Gómez-Esteban et al. 2007; 
Ondo et al. 2002). Auch ein Folsäure- oder Vitamin-B12-Mangel kann mit einem RLS assoziiert sein (Patrick 2007).

Auch tritt das RLS bei der Gabe bestimmter Medikamente auf, bzw. wird durch deren Einnahme verstärkt. $\mathrm{Zu}$ diesen Substanzen gehören überwiegend die Dopaminantagonisten wie Metoclopramid und Neuroleptika, tri- bzw. tetrazyklische Antidepressiva, aber auch Opioidantagonisten (Winkelmann et al. 2001). Durch Neuroleptika kann allerdings auch eine Akathisie verursacht werden, die sich von den Symptomen eines RLS nur schwer unterscheiden lässt (Walters et al. 1991).

Zu einer auffällig häufigen Induktion des RLS scheint es durch Psychopharmaka zu kommen, wie zum Beispiel Clozapin (Duggal und Mendhekar 2007), Lithium (Terao et al. 1991) und Phenytoin (Drake 1988). Allerdings beruhen die Daten hierbei meist auf Einzelfallstudien.

Auf weitere in der Anästhesie typischerweise verwendeten Medikamente wird unter dem Abschnitt 1.2 noch näher eingegangen (siehe auch Tabelle 3).

\subsubsection{Klinik}

Das chronisch verlaufende Krankheitsbild ist motorisch durch Bewegungsdrang und sensorisch durch Parästhesien charakterisiert, die vorwiegend die Beine, aber auch die Arme betreffen können (Michaud et al. 2000; Winkelmann et al. 2000). Ein Teil der Betroffenen beschreibt die Parästhesien als schmerzhaft (Bassetti et al. 2001).

Die Symptome können in ihrer Ausprägung unterschiedlich sein und sind für den Patienten sehr störend. Das RLS kann primär progredient verlaufen, wobei es durch symptomfreie Intervalle (Wochen oder sogar Monate) unterbrochen werden kann. Besonders bei der milderen Form können die Symptome für Jahre ausbleiben (Allen et al. 2003).

Die Einteilung in eine leichte, mittlere und schwere Form erfolgt durch die internationale Schweregradskala für das RLS (IRLS), erstellt durch die International RLS Study Group (IRLSSG, Walters et al. 2003). 
Typisch ist das Auftreten der Symptome in Ruhe bzw. bei Immobilisation (Michaud et al. 2002a; Montplaisir et al. 1998) mit Zunahme in den Abend- und Nachtstunden und einem Maximum an PLMs, einem typischen Befund beim RLS (Trenkwalder et al. 1999a). Auch der Dopamin- und Eisenspiegel befindet sich nun auf seinem tiefsten Niveau (Patrick 2007).

Die Erkrankung zeigt eine zirkadiane Rhythmik, welche sich umgekehrt proportional zur Körpertemperatur verhält (Hening WA et al. 1999; Trenkwalder et al. 1999a). Bei Abnahme der Körpertemperatur in den Abendstunden kommt es zu einer Zunahme der Symptomatik. Die Patienten leiden deshalb an Ein- und Durchschlafstörungen (Allen und Earley 2001; Allen et al. 2003), welche meist der Grund für die Vorstellung beim Arzt sind. Die Patienten mit der schweren Form eines RLS und Schlafstörungen klagen über Tagesmüdigkeit, Antriebslosigkeit, Leistungsabfall und über schlechte Grundstimmung, das heißt, Symptome einer Depression (Winkelmann et al. 2005).

\subsubsection{Diagnostik und Diagnosekriterien}

Das RLS kann aufgrund der Anamnese und der klinischen Symptomatik diagnostiziert werden (Hening W 2004). Zur Orientierung bei der Diagnosestellung dienen die Leitlinien der Deutschen Gesellschaft für Neurologie, basierend auf den von der IRLSSG (Walters 1995) und von Allen et al. (2003) überarbeiteten essentiellen Minimalkriterien (siehe Tabelle 1). Um die Diagnose eines RLS stellen zu können, müssen diese vier Kriterien erfüllt sein.

Ergänzt werden sie durch weitere supportive Kriterien, welche nicht zwangsläufig auftreten, aber häufig mit einem RLS assoziiert sind (siehe Tabelle 2).

Die subjektiv vom Patienten empfundenen Symptome können anhand der IRLS (Walters et al. 2003) beurteilt und in unterschiedliche Schweregrade eingeteilt werden.

Objektive Befunde liefern die beim RLS typischerweise auftretenden, aber nicht obligaten PLMS durch Untersuchungen im Schlaflabor (Montplaisir et al. 1986). 
Zusätzlich können bei RLS-Patienten auch PLMWs beobachtet werden (Hening WA et al. 1986). Die PLMs können ein- oder beidseitig auftreten und zeichnen sich durch die Dorsalflexion des Sprunggelenks aus (Zucconi et al. 2006). Insgesamt stellen sie jedoch ein eher unspezifisches Phänomen dar, das zwar bei 80\% der RLS-Patienten vorkommt, aber auch bei anderen Erkrankungen wie Narkolepsie oder Schlafapnoesyndrom und in bis zu $60 \%$ bei Gesunden in höherem Lebensalter auftritt (Hornyak et al. 2006).

Die neurologische Untersuchung ist beim primären RLS unauffällig. Bei der sekundären Form kommt es je nach Ursache zu pathologischen Veränderungen.

Tabelle 1: Essentielle Kriterien zur Diagnose des RLS (Walters 1995):

1. Bewegungsdrang der Beine oder in selteneren Fällen auch der Arme, ggf. begleitet von Parästhesien

2. Verschlechterung der Symptomatik in Ruhe bzw. bei Immobilität

3. Teilweise oder vollständige Verbesserung der Symptomatik während einer Bewegung

4. Typische zirkadiane Rhythmik mit Zunahme in den Abendstunden und nachts.

Tabelle 2:Supportive Kriterien zur Diagnose des RLS (Walters 1995):

5. Positive Familienanamnese

6. Ansprechen auf dopaminerge Therapie

7. Periodische Beinbewegungen (im Schlaf oder im Wachzustand): PLMS/PLMW

\section{Zusatzuntersuchungen}

Die Zusatzuntersuchungen sind eine Möglichkeit zur weiteren Verifizierung der Diagnose eines RLS, bzw. um andere Diagnosen auszuschließen. 
a) Elektromyographie (EMG) und Elektroneurographie (ENG):

Bei der EMG wird die elektrische Muskelaktivität über konzentrische Nadelelektroden abgeleitet und liefert Informationen über Potentialschwankungen von bestimmten motorischen Einheiten.

Die ENG dient zu Bestimmung des Funktionszustandes eines peripheren Nerven. Abgeleitet werden die Nervenleitgeschwindigkeit, die Amplitude und die Refraktärzeit zum Beispiel des Ulnar- oder Suralisnerven (Tacconi et al. 2004).

Die Nervenleitgeschwindigkeit beim RLS ist unauffällig (Provini et al. 2001).

Diese Untersuchungsmethoden grenzen das RLS zu den PNP ab, welche hier pathologische Veränderungen zeigen würden.

\section{b) Laboruntersuchungen:}

Die Laboruntersuchungen bieten die Möglichkeit, sekundäre Ursachen eines RLS auszuschließen.

Die Bestimmung des Hämoglobins, des mittleren korpuskulären Volumens der Erythrozyten (MCV) und deren mittleres korpuskuläres Hämoglobin ( $\mathrm{MCH}$ ) dient dem Ausschluss einer Anämie, der Ferritinwert liefert Informationen über den Zustand des Eisenspeichers und ist bei Eisenmangel erniedrigt. Auch verminderte Werte von Vitamin B12 und Folsäure bei Verdacht auf Vitaminmangel können Aufschluss über mögliche Ursachen eines RLS geben, bzw. einen Verdacht auf PNP erhärten.

c) Levodopa (L-Dopa)-Test:

Der L-Dopa-Test dient zur ex-juvantibus-Diagnose eines RLS, wenn noch keine dopaminerge Therapie erfolgte bzw. deren Ansprechen nicht eindeutig zu quantifizieren ist (Stiasny-Kolster et al. 2006). Kommt es zu keiner Besserung der Beschwerden, schließt dies ein RLS nicht aus. 
Der Patient bekommt 100mg L-Dopa per os bei Einsetzen der Beschwerden, danach wird das Ansprechen der Therapie mittels der IRLS bestimmt.

\section{d) Polysomnographie (PSG)}

Bei der PSG handelt sich um eine umfangreiche Untersuchungsmethode zur Diagnostik von Schlafstörungen, durchgeführt im Schlaflabor, bei der zum einen ein Elektroenzephalogramm (EEG) und ein Elektrokardiogramm abgeleitet werden, zum anderen Augen- und Beinbewegungen und die Atmung überwacht werden.

Diese Untersuchung kann eventuell bei RLS-Patienten erforderlich sein, um objektiv die Schwere der Schlafstörungen festzustellen und andere Ursachen von Tagesmüdigkeit auszuschließen, wie z. B. das Schlaf-Apnoe-Syndrom.

Es gibt kein typisches Befundmuster der PSG bei RLS-Patienten. Die Schlafqualität ist nachweislich durch eine verminderte Gesamtschlafdauer mit vermehrten PLMs, eine verlängerte Einschlaflatenz und einen erhöhten Anteil an Wachphasen vermindert (Hornyak et al. 2007).

\subsubsection{Differentialdiagnosen}

Es gibt eine Reihe von Differentialdiagnosen, insbesondere aus dem neurologischen Krankheitsspektrum, die vom RLS abgegrenzt werden müssen. Hierbei gibt es sowohl solche Erkrankungen, bei denen eher die Bewegungsstörungen und Schmerzen im Vordergrund stehen, wie z.B. Einschlafmyoklonien, Wadenkrämpfe oder Radikulopathien und solche, welche eher mit Schlafstörungen assoziiert sind, wie das Schlaf-Apnoe-Syndrom oder Depressionen (Winkelmann et al. 2005).

Eine der wichtigsten Differenzialdiagnosen des RLS ist die PNP. Eine genaue Zuordnung kann erschwert sein, da der Patient sowohl Symptome einer PNP als auch eines RLS haben kann. Die PNP kann einerseits das RLS verstärken, andererseits auch die Symptome eines RLS imitieren. Eine genaue Abgrenzung zum RLS ist für die weitere Behandlung wichtig (Allen et al. 2003; Walters 1995). 
Auch die Abgrenzung zur Neuroleptika-induzierten Akathisie, welche ihren Ursprung im extrapyramidalen System hat, ist von Bedeutung. Sie unterscheidet sich von den Symptomen eines RLS dadurch, dass sie mit einem subjektiv empfundenen Bewegungsdrang des ganzen Körpers ohne Missempfindungen einhergeht, und Bewegung kaum Linderung bringt. Es gibt keine zirkadiane Rhythmik, es kommt zu weniger Schlafstörungen und PLMs (Walters et al. 1991).

\subsubsection{Pathophysiologie}

Die Pathophysiologie des RLS ist noch weitgehend unbekannt.

Da betroffene Patienten gut auf eine dopaminerge und opioiderge Therapie ansprechen, ist eine Beteiligung dieser Neurotransmittersysteme wahrscheinlich (Akpinar 1987; Brodeur et al. 1988; Hening W et al. 1999; Walters et al. 1993; Wetter et al. 1999).

Winkelmann et al. (2001) konnten in der Studie zeigen, dass das RLS durch Gabe von Dopamin- oder Opioidantagonisten bei RLS-Patienten nicht provoziert werden kann. Daher ist davon auszugehen, dass es beim RLS durch spezifische neuronale Veränderungen im normalen Ablauf $\mathrm{zu}$ Störungen und damit $\mathrm{zu}$ den krankheitsspezifischen Symptomen kommt, und nicht die allgemeine Verminderung der Neurotransmitter für das Auftreten der Symptome verantwortlich ist.

Möglicherweise könnte auch der Eisenmetabolismus bei der Entstehung des RLS eine Rolle spielen, da Eisen bei der Dopaminsynthese als Kofaktor fungiert, und ein zentraler sowie peripherer Eisenmangel die typischen RLS-Symptome verstärken kann (Allen und Earley 2007). Die Studie von O'Keeffe et al. (1994) zeigte, dass sich bei älteren Patienten der Ferritinspiegel umgekehrt proportional zur Stärke der RLSSymptomatik verhielt: wurde ein niedriger Ferritinspiegel gemessen, hatte der Patient deutlich mehr Beschwerden als bei Normalwerten; nach Eisensubstitution kam es zu einer Besserung der Beschwerden. 
Es konnte noch nicht abschließend geklärt werden, ob es sich beim RLS um eine Erkrankung des peripheren (PNS) oder des zentralen Nervensystems (ZNS) handelt. Vielmehr scheint es zu einer Veränderung der Integration beider Systeme zu kommen, so dass eine eindeutige pathophysiologisch-anatomische Zuordnung schwierig ist. Auch ist kein primär anatomischer Ort bekannt, der für die Erkrankung verantwortlich gemacht werden kann.

Um nun der Erkrankung weiter auf den Grund zu kommen, wurden verschiedene Studien mit unterschiedlichen Überlegungsansätzen und Untersuchungsmethoden rund um das PNS und ZNS durchgeführt, die im Folgenden kurz erläutert werden sollen.

Eine primär kortikale Entstehung kann ausgeschlossen werden, da im EEG und EMG keine Bereitschaftspotentiale in Assoziation mit PLMs abgeleitet werden konnten (Lugaresi et al. 1986; Trenkwalder et al. 1993), und es sich somit um unwillkürliche Bewegungen handelt.

Allerdings konnte im EEG gezeigt werden, dass die sogenannten Beta-Wellen bei RLS-Patienten gesteigert waren, so dass eine erhöhte Erregung des Motorkortex vermutet wird. Man geht beim RLS-Patienten von einer Disinhibition neuronaler Vorgänge aus, die für die typischen Symptome verantwortlich gemacht werden (Schober et al. 2004).

Untersuchungen bei RLS-Patienten mittels transkranieller Magnetstimulation (TMS), mit der die Erregbarkeit des motorischen Systems und das Niveau intrakortikaler inhibitorischer und exzitatorischer Neuronenkreise untersucht werden kann, zeigten unauffällige Werte für die motorische Schwelle sowie für motorisch evozierte Potentiale (Tergau et al. 1999). Tergau et al. konnten in dieser Studie im Gegensatz zur einfachen TMS bei der gepaarten TMS an RLS-Patienten eine signifikante Reduktion der intrakortikalen Inhibition der Fuß- und Handmuskeln feststellen. Sie folgern daraus eine subklinische Disinhibition des Motorkortex bei RLS-Patienten. 
In zwei weiteren TMS-Studien konnte gezeigt werden, dass bei RLS-Patienten eine signifikant kürzere kortikale „silent period“ als bei gesunden Probanden vorliegt (Entezari-Taher et al. 1999). Die "silent periods“ (SP) kommen durch die TMS selbst zustande, da diese intrakortikale inhibitorische Mechanismen induziert: ein weiterer Hinweis für die Übererregbarkeit des Motorkortex.

Hingegen konnte bei mit L-Dopa behandelten Patienten, die initial eine Verkürzung der SP zeigten, nach Einnahme des Medikaments eine Verlängerung der SP des Musculus tibilis anterior gezeigt werden (Stiasny et al. 2003). Auf welcher Ebene sich die inhibitorische Wirkung des L-Dopa abspielt, ob spinal, basalganglionär oder kortikal, ist weiterhin unklar.

Zur weiteren Erforschung des RLS wurden Untersuchungen subkortikaler Regionen mit bildgebenden Verfahren, welche das dopaminerge striatonigrale System, darstellen, durchgeführt.

In nuklearmedizinischen Untersuchungsmethoden wie der Single-Photon-EmissionComputed-Tomography (SPECT) und der Positronen-Emissions-Tomographie (PET) kam man bei der Untersuchung von RLS-Patienten zum Teil zu unterschiedlichen Ergebnissen.

In der SPECT fand sich eine geringfügig reduzierte postsynaptische striatale Bindungskapazität für Dopamin, die auf eine Störung in dieser Einheit hindeutet (Michaud et al. 2002b; Staedt et al. 1995). Andere SPECT-Studien fanden normale striatale Dopamintransportbindungen und Dopaminrezeptordichten (Eisensehr et al. 2001; Tribl et al. 2002).

In der PET wurde einerseits eine minimale Reduktion der 18FDopa-Aufnahme sowohl im Putamen und Caudatum (Turjanski et al.1999), als auch im Nucleus caudatus (Ruottinen et al. 2000) festgestellt. Andererseits ergaben andere Untersuchungen keinen Unterschied in der Bindung von 18FDopa bei gesunden 
Probanden und RLS-Patienten (Trenkwalder et al. 1999b). Inwieweit hier eine Störung vorliegt oder nicht, konnte noch nicht abschließend geklärt werden.

Auch eine strukturelle Läsion des Hirnstammes als Ursache des RLS konnte aufgrund einer unauffälligen exterozeptiven Reflexantwort des Musculus temporalis ausgeschlossen werden, sowie auch in der konventionellen kranialen Magnetresonanztomographie keine strukturellen Veränderungen bei RLS-Patienten festgestellt werden konnten (Bucher et al. 1996).

In der hochauflösenden funktionellen Kernspintomographie, bei der Stoffwechselvorgänge bedingt durch Aktivität sichtbar gemacht werden können, konnten Bucher et al. (1997) während der sensorischen und motorischen Symptomatik des RLS eine bilaterale Aktivierung des Kleinhirns und eine kontralaterale Aktivierung des Thalamus zeigen. Den Untersuchungen zufolge liegt die Vermutung nahe, dass das Zerebellum eine Rolle bei der sensiblen und motorischen Komponente des RLS spielt und möglicherweise der Angriffspunkt der Opiate, die einen wesentlichen Teil der Therapie des RLS darstellen, ist.

Desweiteren gibt es Studien, welche den Ablauf des Flexorreflexes als Modell für die PLM untersuchen. Dieser Schutzreflex der unteren Extremität wird bei Reizung des Nervus plantaris medialis ausgelöst und zeigt bei RLS-Patienten deutlich niedrigere Schwellenwerte als bei gesunden Probanden (Bara-Jimenez et al. 2000). Dies ist eventuell ein Hinweis für spinale Reflexabläufe, die durch die Disinhibition zustande kommen und $z u$ den PLM bei RLS-Patienten führen. Die periphere Nervenleitgeschwindigkeit ist unauffällig (Walters 1995).

Wie die o.g. Untersuchungen zeigen, konnte die Pathophysiologie bislang nicht abschließend geklärt werden. 


\section{Genetik}

Bei mehr als 50\% der idiopathischen RLS-Patienten liegt eine familiäre Belastung vor (Montplaisir et al. 1997; Winkelmann et al. 2000).

Ein eindeutiger Vererbungsmodus konnte bis jetzt nicht gefunden werden. Man geht allerdings von einem autosomal-dominanten Erbgang mit hoher Penetranz aus. Der Erkrankungsbeginn bei der hereditären Form liegt früher als bei der sporadischen Form und eine Antizipation, also ein immer früheres Auftreten der Erkrankung in der jüngeren Generation, wurde deutlich (Lazzarini et al. 1999; Trenkwalder et al. 1996a; Winkelmann und Ferini-Strambi 2006).

Ein RLS-Gen konnte bislang nicht identifiziert werden, allerdings wurden bereits fünf unabhängige chromosomale Regionen durch genomweite Kopplungsuntersuchungen gefunden (Chromosom 12q, 20p, 14q, 9p und2q), auf denen sich möglicherweise das RLS-Gen befindet. Die genetische Heterogenität lässt darauf schließen, dass genetische Varianten in verschiedenen Genen zur Entstehung der Erkrankung beitragen. In genomweiten Assoziationsstudien konnten drei genomische Regionen ausgemacht werden, die als Risikoallele mit einer $50 \%$ Wahrscheinlichkeit zum RLS beim Träger führen (Winkelmann et al. 2006). Welche Voraussetzungen, die zum Ausbruch der Erkrankung führen, gegeben sein müssen, ist allerdings weiterhin unklar.

Die genomischen Regionen kodieren die Gene MEIS1, BTBD9 und MAP2K5/LBXCOR1. Bei homozygoten Trägern aller drei Risikoallele ist das Erkrankungsrisiko um das zwanzigfache erhöht. Überraschenderweise sind die entdeckten Gene in der embryonalen Entwicklung an der Ausbildung der Extremitäten und des zentralen Nervensystems beteiligt. Die Rolle beim Erwachsenen konnte noch nicht abschließend geklärt werden. Durch das Gen MEIS1 wird die Produktion des Neurotransmitters Dopamin steuert und somit indirekt die Bewegung des Körpers kontrolliert (Winkelmann et al. 2007). 


\subsubsection{Therapie}

Bis heute ist keine kausale Therapie des primären RLS bekannt. Sie ist rein symptomatisch und wird vom Leidensdruck des Patienten abhängig gemacht. Es besteht sowohl die Möglichkeit einer medikamentösen als auch einer nichtmedikamentösen Behandlung.

Bei der nicht-medikamentösen Therapie können dem Patienten Maßnahmen, wie z.B. Bewegung durch leichten Sport, Massagen, Wechselbäder und der Verbesserung der Schlafgewohnheiten empfohlen werden. Vermieden werden sollten Alkohol und Koffein (Lutz 1978), da diese den Schlaf negativ beeinflussen können (Paulson 2000).

Ziel der medikamentösen Therapie ist eine Verminderung der Symptome und die Verbesserung der Schlafqualität.

\section{Primäres RLS}

Therapie der ersten Wahl beim primären RLS ist die dopaminerge Medikation (Benes et al. 1999; Earley et al. 1998; Montplaisir et al. 1999; Staedt et al. 1998; Trenkwalder et al. 1995; Walters et al. 1988; Wetter et al. 1999). Fast alle Patienten zeigen einen initial positiven therapeutischen Effekt auf die Gabe von L-Dopa oder niedrigen Dosen von Dopaminagonisten. Dieser Effekt bleibt allerdings nicht immer konstant (Benes et al. 1999; Stiasny-Kolster et al. 2006).

In Deuschland zugelassen für die Therapie des RLS sind L-Dopa in Kombination mit einem Dopamin-Decarboxylase-Hemmer wie Benserazid (Restex $®$, Mardopar ${ }^{\circledR}$ ) oder Carbidopa (Isicom ${ }^{\circledR}$, Nacom ${ }^{\circledR}$ ) und die nicht ergolinen Dopaminagonisten Ropinirol (Adartrel $\circledast$ ) und Pramipexol (Sifrol $\circledast$ ) (Benes et al. 1999; Montplaisir et al. 2000; Trenkwalder et al. 1995; Trenkwalder 2006). Sie beeinflussen sowohl die sensorische als auch die motorische Komponente des Krankheitsbildes positiv. 
Benes (2006) konnte an einer kleinen Gruppe von Patienten zeigen, dass die transdermale Gabe von Lisurid, ebenfalls einem Dopaminagonisten, eine positive Wirkung auf die Symptome hat. Für die Zukunft ist dies sicher eine weitere Therapieoption.

Es liegen außerdem placebo-kontrollierte klinische Studien mit dem Ergot-Dopaminagonisten Cabergolin (Cabaseril $\AA$ ) und Pergolid (Parkotil $₫$ ) vor (Oertel et al. 2006; Stiasny et al. 2000; Trenkwalder et al. 2004), deren Einsatz aber noch nicht zugelassen ist.

In den Studien konnte gezeigt werden, dass die PLMs unter dopaminerger Therapie zurückgingen und eine objektive und subjektive Verbesserung des Schlafes erreicht werden konnte (Benes et al. 1999; Trenkwalder et al. 1995). Allerdings können die Ergot-Dopaminagonisten zu einer Fibrose der Herzklappen oder der Lunge führen, und es können vermehrt Dyskinesien auftreten (Kvernmo et al. 2008).

Die Dosis des zur Behandlung des RLS eingesetzten Medikaments muss individuell auf den Patienten abgestimmt werden.

Nebenwirkungen der Dopaminagonisten sind Übelkeit, Benommenheit und orthostatische Dysregulation, besonders in den ersten vier Wochen der Behandlung, so dass mit niedriger Dosierung begonnen werden und diese bis zur gewünschten Dosis gesteigert werden sollte.

Ihre wichtigste Nebenwirkung ist jedoch die Augmentation, welche besonders unter Therapie mit L-Dopa auftritt und bis zu 60\% aller mit L-Dopa therapierten Patienten betreffen kann (Allen und Earley 1996). Als Augmentation bezeichnet man die nach initialem Therapieerfolg auftretende Verlagerung der Symptome von den Abendstunden auf den Tag, wobei zusätzlich mit einer Zunahme der Beschwerden zu rechnen ist. In diesem Fall ist eine Änderung der Dosierung oder des Einnahmezeitpunktes erforderlich. Oft muss der Dopaminagonist sogar abgesetzt werden. Um das Auftreten dieser Nebenwirkung zu vermeiden, sollte die Dosis 
möglichst niedrig gehalten werden (Paulus und Trenkwalder 2006). Ein möglicher Grund für dieses Phänomen kann ein erniedrigter Ferritinspiegel sein (Trenkwalder et al. 2008a).

Bei Therapieversagen mit oben genannten Medikamenten oder der schmerzhaften Form des RLS, kann eine Therapie mit Opiaten versucht werden, auch wenn diese in der Therapie des RLS nicht zugelassen sind (Walters et al. 1993 und 2001). In der Praxis kommen sie oft zur Anwendung, da sie eine Alternative bei Augmentation bieten. Geeignete Substanzen sind Codein in leichten intermittierenden Fällen, Tramadol (Tramal $\circledast$ ) und Oxycodon (Oxygesic $®$ ) in schwereren Fällen (Karroum et al. 2008). Sie führen zu einer Linderung der sensorischen und motorischen Komponente des RLS und einem erholsameren Schlaf (Walters et al. 1993).

An Nebenwirkungen kann es zu einer Atemdepression und eventuell einem Schlafapnoesyndrom kommen. Das Risiko einer Sucherkrankung wird als gering eingeschätzt, und durch eine alternierende Gabe sind Toleranzeffekte vermeidbar.

Außerdem können Antikonvulsiva wie Carbamazepin (Lundvall et al. 1983; Zucconi et al. 1989), Gabapentin, welches zusätzlich positive Wirkung auf neuropathische Schmerzen hat (Happe et al. 2003) und Valproinsäure (Eisensehr et al. 2004) versucht werden (Trenkwalder et al. 2008b).

Nach der Therapie mit Gabapentin bei RLS-Patienten konnten Garcia-Borreguero et al. (2002) zeigen, dass es zu einer deutlichen Verminderung von PLMS und einer verbesserten Schlafeffizienz kam.

In der klinischen Studie von Conti et al. (2008) wurde die Wirkung einer antikonvulsiven Therapie beim RLS nicht bestätigt.

Auch können Benzodiazepine wie Clonazepam (Boghen 1980; Saletu et al. 2001) versucht werden. In der Therapie des RLS werden sie selten empfohlen, führen aber zu einer Erhöhung der Schwelle für Weckreaktionen und so zu einem für den RLS- 
Patienten erholsameren Schlaf (Saletu et al. 2001), da sie eine hemmende Wirkung auf die beim RLS gesteigerte neuronale Erregbarkeit haben.

Die Antikonvulsiva und Benzodiazepine sind allerdings, genauso wie die Opioide, nur Medikamente zweiter Wahl.

\section{Sekundäres RLS}

Beim sekundären RLS kann eine Behandlung der Grunderkrankung zur Minderung der RLS-Symptomatik führen. Besteht z.B. ein Eisenmangel, kann dieser durch Substitution behoben werden (O'Keeffe et al. 1994). Auch bei einer RLSSymptomatik bei Niereninsuffizienz konnte eine Besserung nach Transplantation beobachtet werden (Kavanagh et al. 2004). Zusätzlich kann unterstützend medikamentös therapiert werden.

Es sollte überprüft werden, ob der Patient ein Medikament einnimmt, das in Verdacht steht, das RLS zu verstärken bzw. auszulösen. Falls möglich, sollte dieses dann abgesetzt werden (siehe Tabelle 3).

\subsection{RLS und Anästhesie}

Wie nun aus den Erläuterungen der vorherigen Kapitel deutlich wurde, sind einige auslösende Faktoren des sekundären RLS bekannt, u.a. auch Medikamente, die in der Anästhesie verwendet werden. Hierzu gehören in erster Linie Neuroleptika mit inrer dopaminantagonistischen Wirkung, insbesondere das Droperidol (INN), das auch als Dehydrobenzperidol (DHB) bekannt ist. Droperidol war ein wesentlicher Bestandteil der sog. Neuroleptanästhesie und wird weiterhin als Antiemetikum der zweiten oder dritten Wahl verwendet. Weitere Medikamente mit dopaminantagonistischer Wirkung sind die Antiemetika Metoclopramid (Paspertin®) und Domperidon, sowie sedierende Neuroleptika wie Promethazin (Atosil囚), die 
gelegentlich zur Prämedikation verwendet werden. Bei den Dopaminantagonisten muss jedoch angemerkt werden, dass sie eine sog. Akathisie mit Bewegungsunruhe verursachen können, deren Symptome von denen eines RLS nicht unterschieden werden können (Walters et al. 1991), so dass der Zusammenhang dieser Pharmaka mit der pathologischen Entität des RLS nicht völlig zweifelsfrei gesichert ist.

Opiatantagonisten, wie Naloxon, werden angeschuldigt, ein RLS auszulösen oder zu verschlechtern (Walters 2002). Bei Patienten mit RLS, deren Symptome mit Opiaten behandelt werden, kann dieses zutreffen, es ist jedoch nicht gesichert, ob Naloxon selbst die Symptome eines RLS auslöst.

Antidepressiva der unterschiedlichen Gruppen, die allerdings in der Anästhesie eher selten verwendet werden, scheinen ebenfalls ein sekundäres RLS auslösen zu können (Cohrs et al. 2008).

Aber aufgrund der Unsicherheit über die genaue Pathogenese des RLS werden viele weitere Pharmaka ohne ausreichende Evidenz beschuldigt, das Restless-LegsSyndrom auszulösen. Patienten werden durch wohlgemeinte "Leitfäden" der unterschiedlichen Selbsthilfegruppen mit Negativlisten versorgt (siehe Tabelle 3), die sie ihren behandelnden Anästhesisten aushändigen sollen. Diese sind in gewissem Umfang hilfreich, da das RLS erstaunlicherweise noch wenig bekannt ist. Diese Listen tragen aber durch die Vermengung von gesicherten Erkenntnissen und Mutmaßungen erheblich zur Verunsicherung bei.

Als Beispiele seien die Pharmaka Enfluran, ein Inhalationsanästhetikum, und Etomidat, ein intravenöses Hypnotikum, genannt, die als kontraindiziert gelten. Bei diesen Pharmaka, wie auch bei einer Reihe anderer, die nicht in die Liste aufgeführt werden, können bei Einleitung bzw. Ausleitung der Anästhesie, sog. myokloniforme 
Bewegungen, v.a. der Beine auftreten (Van Keulen und Burton 2003). Bei Etomidat beruht dieses u.a. auf Enthemmung auf spinaler Ebene (Meinck et al. 1980).

Enfluran, nicht aber das Stereoisomer Isofluran, kann zerebrale Krampfanfälle induzieren. Dieses gilt ebenso für viele andere Anästhetika, wie Propofol (Reddy et al. 1993) oder Sevofluran, die nicht auf der "Negativliste" stehen. Die Pathogenese dieser unwillkürlichen Bewegungen, die von vorübergehendem Zucken bis hin zum Grand-Mal-Anfall reichen, ist nicht an das dopaminerge System gekoppelt, und hat damit kaum etwas mit dem Restless-Legs-Syndrom zu tun (Voss et al. 2008; Modica et al. 1990).

Vor einigen Jahren erschien eine Arbeit, in der auch die Spinalanästhesie als Auslöser des RLS dargestellt wurde (Högl et al. 2002). Da dieser Studie eine geeignete Kontrollgruppe fehlte, sind die Schlussfolgerungen als unzulässig zu betrachten. Dennoch hat die Arbeit für Verunsicherung gesorgt, v.a. weil die Spinalanästhesie für viele Patienten und viele Indikationen das Anästhesieverfahren der Wahl ist. Diese Ergebnisse forderten deshalb eine genauere Überprüfung.

Tabelle 3:Wirkgruppen und Substanzen, die beim RLS-Patienten vermieden werden sollten (aus: „Restless Legs Syndrom- Leitfaden für Anästhesisten“ (Trenkwalder, 2009)):

1.) Neuroleptika (Butyrophenone, Phenothiazine): z.B. Risperidon (Wetter et al. 2002)

2.) Antiemetika mit dopaminantagonistischer Wirkung: z.B. Metoclopramid, Domperidon (JiménezJiménez et al. 1997)

3.) tri- ud tetrazyklische Antidepressiva: z.B. Mianserin (Markkula und Lauerma 1997)

4.) Opiatantagonisten: z.B. Naloxon (Walters 2002) 


\subsubsection{Spinalanästhesie}

Die SPA ist ein sogenanntes rückenmarksnahes Anästhesieverfahren, das auf einer Unterbrechung der Nervenleitung auf spinaler Ebene beruht. Anwendung findet sie vor allem bei Eingriffen unterhalb des Bauchnabels, d.h. bei orthopädischen oder unfallchirurgischen Operationen der unteren Extremität, Operationen des Urogenitaltraktes und gynäkologischen Eingriffen wie z. B. bei der Sectio. Es kommt zu einer Sympathikolyse mit Blockade der Motorik und Sensibilität.

\subsubsection{Geschichtlicher Hintergrund}

Vor mehr als 100 Jahren wurde die erste Punktion des lumbalen Liquorraums zur Liquorentnahme von dem deutschen Internisten und Chirurgen Quinke durchgeführt (Quinke 1891).

Ein weiterer Schritt der Entwicklung folgte im Jahre 1885 durch theoretische und experimentelle Versuche des US-Amerikaners Corning. Er beschrieb die Eingabe von Kokain in rückenmarksnahe Strukturen, wobei eine Wirkung nicht eindeutig belegt ist (Corning 1885). Fast zur gleichen Zeit, im Jahre 1898, wurde die erste erfolgreiche SPA von dem deutschen Chirurgen Bier und seinem Assistenten Hildebrandt in Selbstversuchen mit Kokain beschrieben (Bier 1899).

Anfang des 20.Jahrhunderts etablierte sich die SPA in der Geburtshilfe, wurde dann aber aufgrund sich häufender Todesfälle wieder verlassen und in den 1950er Jahren wieder aufgegriffen. Heute zählt sie zu den etablierten Verfahren in der Geburtshilfe und ist Technik der Wahl bei Hüfttotalendoprothesen und transurethralen Prostataund Blaseneingriffen.

Seit diesen Anfängen kam es zu immer weiteren Entwicklungen und Verbesserungen der verwendeten Materialien und Techniken, so dass es zum Beispiel durch eine dünne atraumatische Nadel zu weniger Verletzungen des umliegenden Gewebes und einem geringeren Prozentsatz an postspinalem Kopfschmerz kommt. 


\subsubsection{Durchführung}

Nach Desinfektion und örtlicher Betäubung der Haut wird die Führungskanüle unter sterilen Bedingungen zwischen dem zweiten und dritten oder dem dritten und vierten Lendenwirbel platziert, und die Injektionskanüle über die Führungskanüle in den Spinalkanal vorgeschoben. Hierbei muss die Dura mater (harte Hirnhaut) penetriert werden. Der korrekte Sitz zeigt sich durch freien Liquorfluss. Das Lokalanästhetikum wird nun in den Intrathekalraum appliziert. Eine Verletzungsgefahr des Rückenmarks durch die Nadel besteht nicht, da es in Höhe des zweiten Lendenwirbelkörpers endet.

Bei dem Lokalanästhetikum handelt es sich entweder um eine iso-, hypo- oder hyperbare Substanz, je nach gewünschtem Wirkort. Liegt dieser im unteren Bereich der Punktionsstelle, würde eine hyperbare Substanz zum Einsatz kommen, d.h. ein Lokalanästhetikum z.B. versetzt mit Glukose. Nach Ablauf der Fixierungszeit ist die Verteilung des Lokalanästhetikums abgeschlossen, und eine Umlagerung des Patienten unproblematisch. Die Wirkung setzt nach wenigen Minuten ein und beginnt mit einem Wärmegefühl der Beine und/ oder des Gesäßes.

Die SPA ist kontraindiziert bei Unverträglichkeiten gegenüber Lokalanästhetika, einer erhöhten Blutungsneigung, sei es erblich oder medikamentös bedingt, und einer Infektion im Bereich der Punktionsstelle. Auch bei hypovolämischen Patienten und solchen mit erhöhtem Hirndruck sollte von einer SPA abgesehen werden.

\subsubsection{Nebenwirkungen}

Obwohl die SPA ein relativ schonender Eingriff ist, können eine Reihe von Nebenwirkungen auftreten. Hierzu gehören in erster Linie als häufigste Komplikation die Hypotonie und Herzrhythmusstörungen (Bezold-Jarisch-Reflex), aber auch Rückenschmerzen, Übelkeit und Erbrechen. 
Postoperativ kann man oft einen postpunktionellen Kopfschmerz und Harnverhalt beobachten. Seltener kommt es zu neurologischen Komplikationen, bedingt durch raumfordernde oder entzündliche Prozesse.

\section{Postpunktioneller Kopfschmerz}

Beim postpunktionellen Kopfschmerz geht man davon aus, dass Liquorverlust für die Symptome verantwortlich ist. Es ist eine unangenehme, aber vollkommen reversible Nebenwirkung der SPA. Meist tritt dieser am zweiten postoperativen Tag auf und kann von Übelkeit, Erbrechen, Nackensteifigkeit, aber auch Sehstörungen und Tinnitus begleitet werden, je nach Reizung der betroffenen Hirnnerven.

\section{Neurologische Komplikationen}

$\mathrm{Zu}$ neurologischen Ausfällen kann es aufgrund direkter Schädigung des Nerven durch die Nadel oder die Toxizität des Lokalanästhetikums kommen. Insgesamt ist dies jedoch selten.

Die Unverträglichkeit gegenüber dem Lokalanästhetikum äußert sich durch das transiente neurologische Syndrom und geht mit symmetrischen in die Beine ausstrahlenden Rückenschmerzen ohne sensorische und motorische Komponente einher. Sie klingen meist ohne Folgen ab.

Anders ist dies beim Cauda-equina-Syndrom, bei dem es durch die Toxizität des Medikamentes zu einer dauerhaften Schwäche der Beine mit Schwierigkeiten beim Wasserlassen und Stuhlgang kommt.

Indirekte Schädigungen der Nerven durch Kompression und somit zu neurologischen Ausfällen können einerseits durch Epiduralhämatome, andererseits durch Abszesse bedingt sein. Eventuell muss eine operative Entlastung vorgenommen werden. Auch eine Radikulitis, Arachnoiditis oder Meningitis gehören ins Spektrum der Komplikationen nach SPA. 
Kommt es bei Durchführung der SPA zu einer Überdosierung des Lokalanästhetikums, droht Bewusstlosigkeit mit Atemstillstand und Kreislaufversagen. Der Patient muss sofort wiederbelebt, beatmet und mit Katecholaminen therapiert werden.

\subsubsection{Allgemeinanästhesie}

Die Allgemeinanäthesie zeichnet sich durch die Ausschaltung des Bewußtseins aus. Bewusstlosigkeit (Hypnose), Analgesie und Muskelrelaxation werden durch Verwendung spezieller Pharmaka wie z.B. Propofol und Etomidat, Opioiden und Mivacurium oder Rocuronium erzielt. Zur Aufrechterhaltung der Narkose werden meist Perfusoren verwendet.

\subsubsection{Lokalanästhesie und periphere Regionalanästhesie}

Die Lokalanästhesie und periphere Regionalanästhesie führen zu einer örtlich begrenzten reversiblen Hemmung der Schmerzempfindung und Motorik einer bestimmten Region des Körpers.

Bei der Lokalanästhesie wird das Medikament in das Gewebe injiziert (Infiltrationsanästhesie). Der Wirkort sind die sensiblen Nervenendigungen. Einsatz findet sie bei kleineren regionalen Eingriffen.

Die periphere Regionalanästhesie führt zur Blockade von einzelnen Nerven oder Nervengeflechten, wie zum Beispiel dem Plexus brachialis oder lumbosacralis (Leitungsanästhesie). 


\section{Fragestellung}

In dieser prospektiven, kontrollierten Studie wurde die Hypothese überprüft, dass die Spinalanästhesie das Restless-Legs-Syndrom auslösen bzw. die Symptome bei Patienten mit vorbestehendem RLS verschlechtern kann.

Da andere bekannte auslösende Faktoren außer der Anästhesie in der perioperativen Phase zum Tragen kommen können, wurden Patienten, die in Allgemeinanästhesie oder peripherer Regionalanästhesie operiert wurden, als Kontrollen in die Studie eingeschlossen. 


\section{Patienten und Methoden}

\subsection{Patienten}

\subsubsection{Einschlusskriterien}

In die Studie eingeschlossen wurden Männer und Frauen im Alter zwischen 18 und 90 Jahren, die sich einer elektiven Operation im Universitätsklinikum Göttingen oder dem evangelischen Krankenhaus Weende unterzogen und entweder eine Allgemeinanästhesie oder eine SPA bekamen. Sie mussten bereit sein, sich insgesamt dreimal, auch telefonisch, befragen zu lassen.

\subsubsection{Ausschlusskriterien}

Von der Studie ausgeschlossen wurden Patienten, die eine Teilnahme ablehnten, telefonisch nicht erreichbar oder nicht in der Lage waren, die Fragen zu verstehen und adäquat zu beantworten.

Von vornherein ausgeschlossen wurden auch Patienten mit dauerhafter Opiattherapie oder mit neurologischen Erkrankungen, die mit einer Polyneuropathie einhergehen.

\subsection{Untersuchungsinstrument}

\subsubsection{Interviews}

Nach Genehmigung der Studie durch die Ethikkommission, konnte mit der Befragung der Patienten begonnen werden.

Die vermeintlich in Frage kommenden Patienten wurden anhand des Vorplanes für die am nächsten Tag geplanten Operationen des Universitätsklinikums Göttingen und des evangelischen Krankenhauses Weende aufgesucht. Befragt wurden 
Patienten, bei denen eine Allgemeinanästhesie oder SPA geplant war. Die Befragung erfolgte bei allen Patienten durch ein und dieselbe Person von April 2004 bis März 2006.

Nach Aktendurchsicht, um von vornherein einen späteren Ausschluss zu vermeiden, wurden die Patienten gefragt, ob sie bereit wären, an einer Studie teilzunehmen.

Um den Patienten nicht von vornherein auf die Beinsymptomatik zu sensibilisieren und so möglichst wahrheitsgetreue Antworten auf die RLS-Fragen zu bekommen, wurde der Schwerpunkt auf Schlafstörungen gelegt und dem Patienten gesagt, man wolle mit der Studie den Zusammenhang von Narkosen mit Schlafstörungen untersuchen. Dem Patienten wurde erklärt, dass es sich um insgesamt drei Befragungen handele, von denen eine vor der Operation, eine nach einer Woche und die letzte vier Wochen nach der Operation stattfindet, um eine eventuelle Veränderung des Schlafverhaltens feststellen zu können. Der Patient musste damit einverstanden sein, $\mathrm{zu}$ Hause angerufen $\mathrm{zu}$ werden und die Probandeneinverständniserklärung unterschreiben. Jedem Patienten wurde eine Probandeninformation ausgehändigt.

Die Patienten konnten jederzeit ohne Angaben von Gründen aus der Studie austreten.

Notiert wurden zunächst Datum der Befragung, Name, Telefonnummer, Grund der stationären Aufnahme, Gewicht, Größe, Alter, Geschlecht, Vorerkrankungen, Dauermedikation, geplante Behandlung, Rauchergewohnheiten und bei Frauen eine eventuelle Schwangerschaft.

Im Anschluss daran wurde zusammen mit dem Patienten der Fragebogen beantwortet. Dies dauerte im Schnitt 5 bis 10 Minuten bei Erstbefragung. Die zweite und dritte Befragung dauerten in der Regel kürzer, wobei sie meist, bedingt durch die Entlassung des Patienten, telefonisch durchgeführt wurden. 
In den darauf folgenden Tagen wurde laut Narkoseprotokoll die Art der Narkose, die verwendeten Anästhetika, die Operationsart und ihre Dauer, die postoperative Medikation, ggf. perioperative Komplikationen, postoperative Mobilisation, Verweildauer des Patienten und Laborwerte (wie Hämatokrit, $\mathrm{MCV}, \mathrm{MCH}$, Schilddrüsenwerte, Eisen, Kreatinin), falls vorhanden, notiert.

In einem Kalender wurden Name und die Telefonnummer des Patienten vermerkt, wobei sogleich eine Woche und vier Wochen später die Notiz wiederholt wurde, um den Fragebogen zum gegebenen Zeitpunkt erneut mit dem Patienten zu besprechen. Durch farbliche Kennzeichnung war stets zu ersehen, um welche Befragung es sich bei dem jeweiligen Patienten handelte.

Patienten, die die RLS-spezifischen Fragen mit "ja“ beantworteten, wurden bei der letzten Befragung darauf hingewiesen, dass sie in nächster Zeit erneut von Kollegen aus der klinischen Neurophysiologie angerufen würden, um die Symptome weiter zu spezifizieren und eventuell Rückschlüsse auf das Vorliegen des RLS schließen zu können.

Da diese Befragungen über einen längeren Zeitraum erfolgten, wurde der erste Teil der Studienpatienten von Frau Dr. med. S. Happe betreut, der zweite Teil von Herrn Dr. med. Dr. rer. nat. J. Schindehütte (beide klinische Neurophysiologie). Ihnen wurde eine Liste mit Patienten ausgehändigt, welche RLS-typische Symptome bei der Befragung äußerten. Diese Patienten wurden erneut telefonisch kontaktiert und durch speziell auf das RLS abgestimmte Fragen konnte geklärt werden, ob es sich bei den angegebenen Symptomen um das RLS handelte oder nicht.

Anhand dieser Daten errechnet sich der Anteil der in dieser Studie rekrutierten RLSPatienten.

Verwaltet wurden alle Daten mittels Tabellenkalkulation. 


\subsubsection{Fragebogen}

Erstellt wurde der Fragebogen in Zusammenarbeit mit den Kollegen aus der klinischen Neurophysiologie (siehe Anhang).

Inhaltlich kann dieser in drei Abschnitte unterteilt werden, in denen der Patient einerseits $\mathrm{zu}$ seiner psychischen Verfassung und seinem Schlafverhalten, andererseits zu typischen Beschwerden eines RLS befragt wird.

Dem Fragebogen zugrunde liegen wissenschaftlich anerkannte Tests, Studien bzw. Leitlinien.

Um einen Überblick über die Gemütslage des Patienten zu erlangen, wurden Fragen und Aussagen, bei denen dieser eine Selbsteinschätzung vornehmen musste, aus dem Center for Epidemiologic Studies Depression Scale (CES-D Scale, Radloff 1977) verwendet.

Die Fragen zum Schlaf des Patienten entstammten sowohl dem Pittsburgh Schlafqualitätsindex (PSOI, Buysse et al. 1989), welcher retropektiv über einen Zeitraum von vier Wochen die Schlafqualität erfragt, als auch der Epworth Sleepiness Scale (ESS, Johns 1991) zur Erfassung der Tagesschläfrigkeit in typischen Alltagssituationen.

Die Fragen zum RLS wurden der IRLS, erstellt durch die IRLSSG zur Erfassung des Schweregrades des RLS (Walters et al. 2003), entnommen und an das Ende des Fragebogens gestellt.

Die beiden ersten Abschnitte des Fragebogens dienten in erster Linie dazu, den Patienten, wie zuvor angekündigt, zu seinen Alltags- und Schlafgewohnheiten zu befragen, um seine Aufmerksamkeit nicht von vornherein auf die für das RLS typische Beinsymptomatik zu lenken und dadurch möglicherweise nicht der Wahrheit entsprechende Antworten zu erhalten (Furnham 1986). 


\section{Fragen zur Depression}

Begonnen wurde der Fragebogen mit zehn Aussagen, zu denen der Patient sich selbst in einer Skala von „kaum oder nicht“ bis hin zu "meistens oder die ganze Zeit“, bezogen auf die vergangene Woche, einschätzen musste. Durch Zahlen von null bis drei wurde eine Art Punktevergabe simuliert. Aus diesem Teil des Fragebogens ergab sich ein erster Eindruck über die Gemütslage des Patienten.

Es folgten fünf Fragen aus dem Alltag, die nur mit „ja“, „nein“ oder "manchmal“ beantwortet werden konnten und eine Aussage über die Gewohnheiten des Patienten zuließen. Besonderes Augenmerk wurde dabei auf sitzende Tätigkeiten wie Fernsehen, Theater, Reisen mit verschiedenen Verkehrsmitteln gelegt. Situationen, die der RLS-Patient möglichst meidet.

\section{Fragen zur Schlafqualität}

Im zweiten Teil wurde der Patienten in zwölf Fragen zu seinen Schlafgewohnheiten und zu seinem subjektiven Empfinden über dessen Qualität befragt. Als Zeitraum wurden die vergangenen vier Wochen gewählt. Der Patient musste ungefähr abschätzen, wie oft er in diesem Zeitraum an Schlafstörungen aufgrund unterschiedlicher Ursachen litt (z.B. Ein- oder Durchschlafschwierigkeiten, Nykturie, Dyspnoe). Als Antwortmöglichkeiten standen "drei oder mehr pro Woche“, „ein- bis zweimal pro Woche“, „weniger als einmal pro Woche“ oder "in den letzten vier Wochen gar nicht" zur Auswahl.

\section{Fragen zum RLS}

Die für die Selektion der RLS-Patienten entscheidenden Fragen schlossen sich der Flut für die Studie weniger relevanten Fragen an. Hierbei wurde der Patient gefragt, ob er bei sich je Missempfindungen (Kribbeln, Ameisenlaufen oder Schmerzen) an den Beinen verbunden mit einem Bewegungsdrang beobachtet hätte. Wurde diese 
Frage mit nein beantwortet, war die Befragung beendet, wurde sie bejaht, wurde durch die anschließenden Fragen versucht, dieses Missempfinden zu konkretisieren, bzw. typische für das RLS auftretende Befunde zu erheben: Ein- und Durchschlafstörungen, Zunahme der Beschwerden in den Abendstunden und in Ruhe, Tagesmüdigkeit, allgemeine Abgeschlagenheit und eine positive Familienanamnese.

\subsection{Anästhesieverfahren}

\subsubsection{Spinalanästhesie}

Nach Desinfektion und Aufbringen eines sterilen Tuches auf die untere Rückenpartie des sitzenden Patienten, wurde in Höhe der Beckenkämme nach den Dornfortsätzen getastet, um nach Setzen der Lokalanästhesie mit Mepivacain 1\%, zwischen dem zweiten und dritten oder dem dritten und vierten Lendenwirbelkörper mit der atraumatischen Führungskanüle den Bandapparat zu penetrieren. Durch die Führungskanüle wurde nun die eigentliche Punktionsnadel mit einer Dicke von 27, 25, unter anatomisch schwierigen Gegebenheiten auch 22 Gage, durch die Dura mater bis in den Subarachnoidalraum vorgeschoben. Liquorfluss zeigte den richtigen Sitz an. Das Lokalanästhetikum Bupivacain 0,5\% hyperbar wurde in der für den Eingriff erforderlichen Menge von 2,0 bis 5,0ml appliziert, und der Patient mit erhöhtem Oberkörper gelagert.

\section{Bupivacain}

Bupivacain besitzt von allen Lokalanästhetika die höchste Toxizität, wenn es in zu großen Mengen in den systemischen Kreislauf gelangt. Die kardialen Symptome können bei dieser Substanz auch vor den ZNS-Symptomen auftreten. 


\subsubsection{Allgemeinanästhesie}

Nach Legen eines peripheren Zugangs, Anlegen des EKGs und Oxymeters, wurde der Patient für einige Zeit mit Sauerstoff bebeutelt. Dann bekam er über die Vene das Hypnotikum, entweder Propofol oder Etomidat, und das Opioid, bei Intubation zusätzlich auch ein Muskelrelaxans.

Die Anästhesie wurde durch die kontinuierliche Gabe einer hypnotisch wirksamen Substanz (in den meisten Fällen Propofol), sowie eines Opioids aufrechterhalten. Die Zufuhr der Medikamente wurde kurz vor Operationsende beendet.

\subsubsection{Lokalanästhesie und periphere Regionalanästhesie}

Die Lokalanästhesie und periphere Regionalanästhesie fanden unter sterilen Bedingungen statt.

Die Lokalanästhesie wurde durch Infiltration des zu betäubenden Gewebes mit Mepivacain 2\% durchgeführt. Da es fast ausschließlich Patienten aus der Augenklinik waren, handelte es sich in den meisten Fällen um Retrobulbäranästhesien. Die Nadel wurde entlang des Orbitabodens in Richtung des Sehnerven vorgeschoben und ungefähr $5 \mathrm{ml}$ Mepivacain appliziert.

Bei der peripheren Regionalanästhesie wurde der Nerv oder das Nervengeflecht an der oberen oder unteren Extremität mit einer elektrischen Sonde aufgespürt, und Prilocain 1\% (ca. 40ml) in diesem Bereich in die Tiefe des Gewebes in ausreichender Menge eingespritzt, um den gewünschten Teil der Extremität zu betäuben.

\section{Prilocain}

Prilocain hat die geringste Toxizität und eine ausgeprägte sensorische und nur gering motorische Wirkung. Kombiniert wurde es bei den Studienpatienten mit 
Ropivacain, das dem Bupivacain ähnlich ist und zudem eine höhere analgetische Potenz und Wirkdauer als Prilocain aufweist.

\subsection{Statistische Auswertung}

Die Eingabe und Auswertung der ermittelten Daten erfolgte mit dem Statistikprogramm Statistica (Version 8.0, STATSOFT).

Alle ermittelten Werte wurden in eine Datenbank eingegeben und die Ergebnisse der Gruppen nach Studienende gegenübergestellt.

Quantitative Unterschiede zwischen den Gruppen wurden mit dem Kruskal-WallisTest und dem Mann-Whitney-U-Test getestet, qualitative Unterschiede wie die Prävalenz des RLS je nach Geschlecht und Anästhesieform mit dem Chi-QuadratTest (exakter Test nach Fisher, zweiseitig). Ebenfalls zum Einsatz kam dieser Test bei der Beurteilung des Neuauftretens eines RLS bzw. seiner Verschlechterung durch die jeweilige Narkose. Die maximale Wahrscheinlichkeit eines postoperativ auftretenden RLS oder dessen Verschlechterung wurde für das $99 \%$ Konfidenzintervall berechnet.

Das Signifikanzniveau wurde auf $p$ kleiner 0,05 festgelegt. 


\section{Ergebnisse}

Insgesamt wurden 398 Patienten in die Studie aufgenommen. Keiner der Befragten, unabhängig von der Art der Narkose, entwickelte ein RLS, noch verstärkten sich die Symptome bei bereits bestehender Symptomatik.

\subsection{Demographische Daten des Patientenkollektivs}

Von den 398 Patienten waren 227 Männer und 171 Frauen. Keine der Frauen war zum Zeitpunkt der Befragung schwanger.

Zweihundertzwölf Patienten bekamen eine Allgemeinanästhesie und 147 eine SPA. Bei den verbliebenen 39 Patienten kam es kurz vor Operationsbeginn zur Änderung des geplanten Narkoseverfahrens. Ihre Oparation wurde in Lokalanästhesie oder peripherer Regionalanästhesie durchgeführt. Da es sich bei letzteren um ein kleines Patientenkollektiv handelt, erfolgt die Auswertung nur im Hinblick auf das erstmalige Auftreten oder die Verschlechterung eines bereits bestehenden RLS. Das Hauptaugenmerk bei der Auswertung wird im Folgenden hauptsächlich auf die beiden Gruppen der Allgemeinanästhesie und SPA gelegt.

Ein Eisenmangel als Ursache des RLS war bei allen Patienten unwahrscheinlich, da keiner der Patienten mit RLS eine Anämie oder ein erniedrigtes MCV aufwies.

Zehn Patienten hatten einen erhöhten Kreatininwert (über 1,4mg/dl), von denen drei an einer chronischen Niereninsuffizienz litten. Allerdings hatte keiner dieser Patienten ein vorbestehendes RLS.

Bei keinem Patienten wurde ein neu aufgetretenes RLS beobachtet.

Bei 38 Patienten bestand aufgrund des präoperativen Fragebogens die Verdachtsdiagnose eines RLS. Hiervon konnte bei 31 Patienten $(7,8 \%$ aller Patienten), zehn Männern (4,4\% der männlichen Patienten) und 21 Frauen (12,3\% der weiblichen Patienten), die Diagnose durch die neurologische Untersuchung 
bestätigt werden. Keiner dieser Patienten berichtete von einer Verschlechterung nach dem operativen Eingriff.

\subsubsection{Gruppe der Patienten mit Spinalanästhesie}

Insgesamt wurde bei 147 Patienten (84 Männer, 63 Frauen) eine SPA durchgeführt. Das Durchschnittsalter lag bei $61,6 \pm 12,8$ Jahren. Zwölf Patienten $(8,2 \%)$, vier Männer und acht Frauen, hatten ein vorbestehendes RLS. Die SPA wurde bei allen Patienten mit Bupivacain 0,5\% durchgeführt. Verwendet wurden Nadeln mit 25, 27 oder 22 Gauge $(111,33,3)$. Prämediziert wurden die Patienten mit Midazolam, Diazepam und Norazepam (64, 50, 35).

Zehn Patienten musste zusätzlich Propofol zur Sedierung verabreicht werden, da die Operation länger als geplant andauerte, und die Wirkung der SPA langsam nachließ. Von diesen Patienten bekam einer zusätzlich Sufentanil und einer Ketamin.

Bei fünf Patienten wurde eine zusätzliche Atropinmedikation bei bradykarder Arrhythmie (Bezold-Jarisch-Reflex), eine typische Nebenwirkung bei SPA, notwendig. Von diesen Patienten bekam einer zusätzlich Sufentanil, einer Alfentanil und einer Ketamin.

Insgesamt wurden vier Patienten mit Ketamin und sechs Patienten mit Opioiden (Sufentanil 3, Alfentanil 2, Fentanyl 1) versorgt. Vier Patienten erhielten zusätzlich Clonidin. Bei drei Patienten musste eine hypotone Kreislaufsituation mit Theodrenalin und Cafedrin (Akrinor $\left.{ }^{\circledR}\right)$ behandelt werden.

Die postoperative Schmerzmedikation bestand vorwiegend aus Diclofenac und Metamizol (57, 44). Es wurden aber auch Piritramid und Paracetamol (12, 5) verabreicht. Einige Patienten benötigten mehrere Substanzen, andere gar keine. Keinem der Patienten mußte ein Corticoid gegeben werden.

Ein Patient klagte postoperativ über Übelkeit und Erbrechen, welche mit Dolansetron behandelt wurden.

Keiner der Patienten entwickelte einen postspinalen Kopfschmerz. 
Fünfundachtzig Patienten wurden aus der orthopädischen Abteilung (Hüft-, Knie- und Fußoperationen) rekrutiert, 59 Patienten aus der Urologie (transurethrale Blasenund Prostataoperationen, Epididymektomie, Weitung von Ureterstenosen, Lithotripsie) und drei aus der Allgemeinchirurgie (Leistenhernien oder Hämorrhoidenoperationen).

Die durchschnittliche Operationsdauer betrug $56 \pm 34$ Minuten.

\subsubsection{Gruppe der Patienten mit Allgemeinanästhesie}

Insgesamt bekamen 212 der befragten Patienten eine Allgemeinanästhesie.

Das Einleitungsmedikament hierbei war in 209 Fällen Propofol, in drei Fällen Etomidat, in Kombination mit einem Opioid.

Es waren 121 Männer und 91 Frauen mit einem Durchschnittsalter von 44,6 $\pm 16,7$ Jahren. Präoperativ wurde bei 15 Patienten (7,1\%), fünf Männern und zehn Frauen, ein RLS diagnostiziert.

Zur Prämedikation bekamen die Patienten in der Regel Midazolam, gelegentlich Temazepam oder Diazepam (200, 7 bzw. 4 Patienten). Die Beatmung erfolgte über einen Endotrachealtubus (167 Patienten) oder eine Larynxmaske (45 Patienten). Das verwendete Opioid war in den meisten Fällen Remifentanil, seltener Fentanyl, Alfentanil oder Sufentanil (172, 28, 9, 3). Zur Muskelrelaxation wurden Mivacurium bzw. Rocuronium verwendet (42, bzw. 125). Patienten, die über eine Larynxmaske beatmet wurden, bekamen kein Muskelrelaxans. Die postoperative Schmerzmedikation bestand vorwiegend aus Paracetamol intravenös (157), Piritramid (79), Diclofenac (67), Pethidin (4) und Tramadol (2), wobei einigen Patienten mehrere Substanzen verabreicht werden mußte.

Achtzehn Patienten bekamen Atropin, 16 zur Therapie einer Bradykardie und zwei zusammen mit Neostigmin zur Antagonisierung der Muskelrelaxation. Bei einem Patienten musste der Opioidantagonist Naloxon bei anhaltender Atemdepression verabreicht werden. Neunzehn Patienten bekamen postoperativ ein 
Cortisonpräparat, fast alle intravenös Antibiotika zur Infektionsprophylaxe. Neun Patienten entwickelten postoperativ behandlungsbedürftige Übelkeit und Erbrechen, welche mit Dolasetron (7) bzw. Dimenhydrinat (2) behandelt wurde. Keiner der Patienten bekam Droperidol. Bei 46 Patienten wurden orthopädische Eingriffe durchgeführt (Wirbelsäulenoperationen bei Frakturen, Operationen bei Frakturen der Extremitäten, Hüft- und Knieoperationen), 162 Patienten wurden aus der Hals-Nasen-OhrenHeilkunde rekrutiert (Operation bei Septumdeviation, Tonsillektomie, Operationen der Nasennebenhöhlen, laserchirurgische Eingriffe des Larynx, Neckdissection) und drei der Patienten mit Allgemeinanästhesie waren aus der Augenheilkunde (Kataraktextraktion, Basaliomentfernung).

Die durchschnittliche Operationsdauer betrug 122 \pm 75 Minuten.

Tabelle 4: Biometrische und demographische Charakteristika der Patienten mit SPA und Allgemeinanästhesie:

(Mittelwerte \pm Standardabweichung)

\begin{tabular}{|l|l|l|l|}
\hline & $\begin{array}{l}\text { Spinalanästhesie } \\
(\mathrm{n}=147)\end{array}$ & $\begin{array}{l}\text { Allgemeinanästhesie } \\
(\mathrm{n}=212)\end{array}$ & $\mathrm{p}$-Wert \\
\hline Alter (Jahre) & $61,6 \pm 12,8$ & $44,6 \pm 16,7$ & $\mathrm{p}<0.01$ \\
\hline Gewicht (kg) & $78,9 \pm 16,7$ & $82,2 \pm 19,6$ & $\mathrm{p}<0.05$ \\
\hline Größe (cm) & $173 \pm 9,4$ & $172 \pm 8,8$ & n. s. \\
\hline $\begin{array}{l}\text { Geschlechtsvertei- } \\
\text { lung (M / F) }\end{array}$ & $84 / 63$ & $120 / 91$ & n. s. \\
\hline $\begin{array}{l}\text { Operationsdauer } \\
\text { (min) }\end{array}$ & $56 \pm 34$ & $122 \pm 75$ & $\mathrm{p}<0.01$ \\
\hline $\begin{array}{l}\text { Patienten mit RLS } \\
\text { (M / F) }\end{array}$ & $12(5 / 8)$ & $15(5 / 11)$ & n. s. \\
\hline
\end{tabular}

(n.s.= nicht signifikant) 
Wie aus den Ergebnissen in Tabelle 4 ersichtlich ist, unterscheiden sich die beiden untersuchten Gruppen signifikant im Bezug auf Alter der Probanden und Dauer der Operation. Die Patienten, die eine SPA bekommen haben, waren deutlich älter und die Operationsdauer war kürzer als bei der Patientengruppe mit Allgemeinanästhesie. Ein geringfügiger, aber signifikanter Unterschied ergab sich in Hinblick auf das Gewicht der Probanden, wobei die Gruppe der SPA-Patienten durchschnittlich leichter war. Keinen Unterschied gab es im Vergleich der Körpergröße.

Auch konnte im Hinblick auf die Geschlechterverteilung kein signifikanter Unterschied ermittelt werden.

Die maximale Wahrscheinlichkeit, dass sich perioperativ ein RLS erstmals manifestiert bzw. verschlechtert, liegt in dem Gesamtkollektiv der 398 Patienten bei null bis $1,3 \%$. Für die Gruppe mit Allgemeinanästhesie liegt dieser Wert bei null bis $2,7 \%$ und für die SPA bei null bis 4,0\% $(p=0.94)$. Betrachtet man die Gruppen einzeln, liegen die Werte aufgrund der kleineren Gruppengröße höher als in der Gesamtgruppe.

\subsubsection{Gruppe der Patienten mit Lokalanästhesie oder peripherer Regionalanästhesie}

Insgesamt 39 Patienten haben entweder eine Lokal- (28) oder periphere Regionalanästhesie (11) bekommen. Das Durchschnittsalter lag bei $63,3 \pm 16,1$ Jahren. Von den 39 Personen waren 18 Frauen und 21 Männer. Vier Patienten $(10,3 \%)$-ein Mann und drei Frauen- hatten bereits präoperativ ein RLS.

Zwei Patienten bekamen zusätzlich Propofol, einer von innen auch Remifentanil, da die lokale Schmerzauschaltung unzurechend war.Bei den Operationen handelte es sich um Kataraktextraktionen (Augenheikunde) und eine Leberbiopsie (Gastroenterologie). 
Von insgesamt zwei Patienten mit peripherer Regionalanästhesie, die Ketamin bekamen, wurden einem zusätzlich Remifentanil und Propofol wegen Unruhe durch Schmerzen verabreicht. Keiner der Patienten musste aufgrund einer postoperativ aufgtretenden Übelkeit behandelt werden.

Diese Patientengruppe unterzog sich orthopädisch-unfallchirurgischen Operationen (Metallentfernungen, Handoperationen, Operationen von Ligamentverletzungen am Fuss).

Die Operationsdauer betrug $57 \pm 40,6$ Minuten.

\subsubsection{Patienten mit RLS}

In dem gesamten Patientenkollektiv von 398 Probanden befanden sich 31 Personen (7,8\%), bei denen die Minimalkriterien zur Diagnosestellung des RLS, unabhängig davon, ob es sich um die primäre oder sekundäre Form handelte, aus neurologischer Sicht erfüllt wurden. Es waren 10 Männer (32,3\%) und 21 Frauen $(67,7 \%)$. Vier Männer und 10 Frauen klagten zusätzlich über Schlafstörungen. Bei insgesamt neun Patienten konnte eine positive Familienanamnese erhoben werden; acht Patienten konnten keine Angabe zu dieser Frage machen. Drei der 21 Frauen waren kinderlos, drei hatten ein Kind, sieben zwei Kinder, fünf drei Kinder und drei vier oder mehr Kinder (siehe Tabelle 5).

Tabelle 5: Anzahl der Kinder der 21 RLS-positiven Frauen:

\begin{tabular}{|l|l|l|l|l|l|}
\hline $\begin{array}{l}\text { Anzahl der } \\
\text { Kinder }\end{array}$ & 0 & 1 & 2 & 3 & 4 oder mehr \\
\hline $\begin{array}{l}\text { Anzahl der } \\
\text { Frauen }\end{array}$ & 3 & 3 & 7 & 5 & 3 \\
\hline $\begin{array}{l}\text { Angabe in } 14,3 \% \\
\text { Prozent (\%) }\end{array}$ & $14,3 \%$ & $33,3 \%$ & $23,8 \%$ & $14,3 \%$ \\
\hline
\end{tabular}


Die Prävalenz des RLS stieg in dem Patientenkollektiv mit zunehmendem Alter (siehe Tabelle 6). Die typischen Beschwerden bestanden schon vor dem operativen Eingriff.

Drei Patienten wurden mit einem Dopaminpräparat behandelt, zwei von ihnen wegen des RLS, einer aufgrund eines Morbus Parkinson.

Insgesamt neun Patienten litten an einer mit L-Thyroxin behandelten Hypothyreose, drei Probanden gaben als weitere Allgemeinerkrankungen Depressionen an, zwei einen insulinabhängigen Diabetes mellitus und drei ein Schlafapnoesyndrom. Diese nutzten zur Nacht ein CPAP-Gerät (Continuous Positive Airway Pressure, kontinuierlicher Atemwegsüberdruck).

Keiner der RLS-Patienten litt an einer hypochromen mikrozytären Anämie oder einer dialysepflichtigen Niereninsuffizienz.

Tabelle 6: Altersabhängige Prävalenz vom RLS bei 398 operativ versorgten Patienten mit Allgemeinanästhesie, SPA, Lokalanästhesie und peripherer Regionalanästhesie:

\begin{tabular}{|l|l|l|l|}
\hline Altersgruppe & Anzahl der Patienten & Patienten mit RLS & $\%$ \\
\hline $18-20$ & 10 & 0 & 0 \\
\hline $21-30$ & 46 & 1 & 2,2 \\
\hline $31-40$ & 44 & 2 & 4,6 \\
\hline $41-50$ & 60 & 7 & 11,7 \\
\hline $51-60$ & 77 & 9 & 11,7 \\
\hline $61-70$ & 88 & 9 & 10,2 \\
\hline $71-80$ & 57 & 3 & 5,3 \\
\hline $81-90$ & 16 & 0 & 0 \\
\hline
\end{tabular}




\section{Diskussion}

Obwohl das RLS seit mehreren hundert Jahren eine bekannte chronische neurologische Erkrankung ist, die bei bis zu 10\% der Gesamtbevölkerung gefunden werden kann (Berger et al. 2004), ist sie insgesamt eine sehr wenig bekannte und erforschte Erkrankung, deren Bedeutung lange Zeit unterschätzt wurde und welche besonders in den letzten Jahrzehnten immer mehr in den Fokus der Aufmerksamkeit rückt (Högl et al. 2005).

Dass die SPA in 8\% der Fälle einer der auslösenden Faktoren des RLS sein soll, wie es in der Studie von Högl et al. (2002) beschrieben wird, scheint auf den ersten Blick unwahrscheinlich, da die Schlussfolgerung daraus wäre, dass eine scheinbar sehr häufig auftretende Komplikation eines Narkoseverfahrens über einen so langen Zeitraum übersehen worden ist.

Die SPA ist ein seit hundert Jahren angewandtes Standardverfahren in der Anästhesie, und es liegen keine weiteren Studien vor, die einen ähnlichen Zusammenhang von SPA und RLS beschreiben.

Grund hierfür könnte sein, dass weder der ärztliche Kollege, noch der Patient selbst postoperativ aufgetretende RLS-typische Symptome mit der SPA in Zusammenhang gebracht hat, und dieser erst durch genaueres Erfragen mit standardisierten Fragebögen aufgefallen wäre.

In der vorliegenden Arbeit sollte der Einfluss verschiedener Anästhesieformen auf das RLS untersucht werden. Hierzu wurden Patienten, die entweder eine Allgemeinanästhesie oder eine SPA bekamen, einer präoperativen und zwei postoperativen Befragungen mit einem standardisierten Fragebogen unterzogen und beobachtet, ob es zu einer Exazerbation bereits bestehender Symptome oder zu einem erstmaligen Auftreten eines RLS kam. In die Studie aufgenommen wurden neben Patienten mit SPA auch solche mit Allgemeinanästhesie, um ggf. andere Faktoren wie das chirurgische Trauma mit lokalen Entzündungsreaktion (Nemeth 
und Ganz 2006) oder die allgemeine Umstellung während des Krankenhausaufenthaltes mit Immobilisation (Michaud et al. 2002a), die möglicherweise einen Einfluss auf die Entstehung des RLS haben, mit in Betracht zu ziehen.

Nach Auswertung der Daten konnte gezeigt werden, dass in keiner der beiden Patientenkollektive das RLS perioperativ ausgelöst oder verstärkt wurde, und somit die Ergebnisse der Studie von Högl et al. (2002) nicht erhärtet werden konnten.

Im Folgenden sollen die Resultate mit der bereits veröffentlichten Studie verglichen werden, um Aufschluss über Gemeinsamkeiten und Unterschiede zu dieser Studie zu geben, sowie mögliche Einflussfaktoren und Erklärungsansätze für die unterschiedlichen Ergebnisse zu liefern, da sich zwischen beiden eine deutliche Diskrepanz zeigt.

Betrachtet man das Patientenkollektiv dieser Studie, stellt man fest, dass es im Hinblick auf die Prävalenz und die Verteilung des RLS repräsentativ ist.

Bei 7,8\% der insgesamt 398 Patienten wurde das RLS diagnostiziert, in der Gruppe der SPA waren es 8,2\%. Dieses Ergebnis deckt sich mit Ergebnissen anderer Studien, in denen von Prävalenzen zwischen 3-10\% ausgegangen wird (Ekbom 1945 und 1960; Lavigne und Montplaisir 1994; Phillips et al. 2000; Rothdach et al. 2000).

Auch wenn im Schnitt in allen Gruppen weniger weibliche Probanden befragt wurden als männliche, wurde das RLS bei Frauen doppelt so häufig diagnostiziert, und eine mit zunehmendem Alter steigende Prävalenz konnte bestätigt werden (siehe Tabelle 6). Diese Ergebnisse stimmen mit Ergebnissen anderer Studien überein (Berger et al. 2004; Zucconi und Ferini-Strambi 2004). Keine der Frauen war zum Zeitpunkt der Befragung schwanger, so dass die Schwangerschaft als Risikofaktor des RLS nicht berücksichtigt werden musste.

Högl et al. (2002) ermittelten in ihrem Kollektiv von 202 Probanden mit SPA bereits bei $20,3 \%$ ein RLS in der Erstbefragung, die, anders als in vorliegender Studie, nicht 
präoperativ, sondern erst 48 bis 72 Stunden in der postoperativen Regenerationsphase stattfand. Die Prävalenz von über $20 \%$ erscheint im Vergleich zu anderen Stichproben sehr hoch und nicht repräsentativ für die Gesamtbevölkerung, zumal dieselben Autoren in einer später durchgeführten Studie zum RLS einen Wert von 10,6\% publizierten (Högl et al. 2005). So wurden zu weiteren Analysen dieser Studie lediglich die 161 Patienten herangezogen, die kein vorheriges RLS aufwiesen. Aus diesem Patientenkollektiv sollen 8,7\% im Mittel nach 7,3 Tagen ein vorübergehendes RLS nach SPA entwickelt haben- auch dies ein beachtlicher Wert.

Ein Erklärungsversuch für die Ergebnisse der o.g. Studie könnte einerseits die Tatsache sein, dass die zweite Befragung nur innerhalb weniger Tage nach der Erstbefragung stattfand, so dass der Patient möglicherweise durch die Art der Befragung auf die Beinsymptomatik "gepolt“ wurde, indem er die unterschiedlich gewichtete Relevanz der einzelnen Fragen unbewußt wahrnahm. Bei diesem Phänomen aus der Psychologie spricht man von „response bias“, welche zu einer Verzerrung von statistischen Erhebungen bei der Auswertung von Fragebögen führt. Der Befragte antwortet bei sich wiederholenden Fragen nicht der Wahrheit entsprechend, sondern nach der seiner Meinung nach erwünschten Antwort des Interviewers (Furnham 1986).

In der Studie von Högl et al. (2002) kann davon ausgegangen werden, dass die Patienten ausschließlich zum RLS und dessen Schwere befragt wurden, und die Antworten durch die Art der Befragung somit beeinflußt wurden. Zudem ist auffällig, dass die meisten Patienten RLS-typische Symptome gehäuft bei der zweiten Befragung äußerten. Näheres zur Durchführung der Befragung wurde nicht erwähnt.

Da für die Diagnose des RLS lediglich vier Kriterien erfüllt sein müssen, wurden in unseren Fragebogen zusätzlich Fragen zum Schlaf und zur Gemütslage der 
Patienten aufgenommen, und die Patienten wurden darüber aufgeklärt, dass es sich bei der Studie um Untersuchungen zum Einfluss der Anästhesie und Operation auf den Schlaf handele. Hierdurch sollte die Stärke der "response bias“ gering gehalten und ein möglichst genaues Ergebnis erzielt werden.

Andererseits muss für die Beurteilung der Ergebnisse der Studie von Högl et al. (2002) auch die Zusammensetzung des befragten Kollektivs, das aus 71,3\% Frauen, von denen über die Hälfte schwanger war, und aus 28,7\% Männern bestand, in Betracht gezogen werden. Aus anderen Studien ist bekannt, dass es in der Schwangerschaft zu einem gehäuft auftretenden RLS kommen kann (Goodman et al. 1988; Manconi et al. 2004). Somit ist davon auszugehen, dass es sich bei dem befragten Patientengut nicht um einen repräsentativen Querschnitt durch die Normalbevölkerung handelt, und dies eine Erklärung für die hohe Prävalenz des RLS liefern könnte.

In der Studie wird außderdem nicht beschrieben, inwieweit die postoperative Übelkeit mit Erbrechen, die in $76 \%$ der Fälle nach SPA und besonders bei Sectiones beobachtet wird (Santos und Datta 1984; Stein et al. 1997), eine Rolle bei den Patienten gespielt hat. Möglicherweise wurden sie mit zu der Zeit standardmäßig bei postspinal auftretender Übelkeit verwendeten Medikamenten Droperidol oder Metoclopramid- beides Dopaminantagonisten- behandelt (Ward 1989; Foster et al. 1996; Barnes et al. 1982). Nebenwirkung dieser Substanzen ist die sogenannte Akathisie, bei der es zu einer Sitzunruhe kommt, und welche oft nicht eindeutig vom RLS differenziert werden kann (Walters et al. 1991). Außerdem kann die Gabe dieser Medikamente auch zu einer Verstärkung der RLS-Symptomatik führen (Winkelmann et al. 2001).

In vorliegender Studie wurden Patienten mit erhöhtem Risiko für postoperative Übelkeit und Erbrechen gemäß der Leitlinien mit einem Cortisonpräparat oder Serotoninantagonisten vorbehandelt, um das Auftreten der oben beschriebenen 
Symptomatik nach der Operation so gering wie möglich zu halten (Rüsch et al. 2005). Kam es dennoch zu Symptomen, waren Medikamente erster Wahl Serotoninantagonisten wie Dolansetron oder Ondansetron oder Antihistaminika wie Dimenhydrinat. Metoclopramid kommt nicht mehr zum Einsatz und Droperidol nur in seltenen Fällen, sollten andere Medikamente keine Wirkung zeigen.

In vorliegender Studie entwickelten 10 Patienten aus den Gruppen der Allgemeinanästhesie und SPA eine behandlungsbedürftige Übelkeit und Erbrechen. Keinem von innen wurde ein Dopaminantagonist verabreicht, so dass ein weiterer Faktor, welcher für eine Verschlechterung oder das neue Auftreten eines RLS nach dem Eingriff hätte verantwortlich sein können, ausgeschlossen werden kann.

Opioide kommen in der RLS-Therapie zum Einsatz und führen zu einer Linderung der Symptome (Hening W et al. 1999). Keiner der Patienten aus vorliegender Studie stand unter dauerhafter Opioidtherapie, so dass ein normalerweise postoperativ aufgetretendes RLS maskiert worden wäre.

Auch wenn bei der Anästhesie Opioide zum Einsatz kommen, ist primär nicht von einer Beeinflussung der RLS-Symptome im Sinne einer Unterdrückung auszugehen, da, je nach verwendeter Substanz, die Wirkdauer zwischen weniger als acht Minuten (Remifentanil) und wenigen Stunden (Sufentanil, Fentanyl) liegt. Außerdem lag das Maximum des postoperativ aufgetretenen RLS in der Studie von Högl et al. (2002) bei 7,3 Tagen ( $95 \%$ Konfidenzintervall 4,0 bis 10,6 Tage), so dass die bei der Operation verabreichten Opioide keinen Einfluss auf die erhobenen Daten hatten.

Der Opioidantagonist Naloxon mußte lediglich einem Patienten in vorliegendem Patientenkollektiv in der Aufwachphase verabreicht werden, da es durch Opioidgabe während der Operation zu einer anhaltenden Atemdepression kam.

Naloxon gilt beim RLS als kontraindiziert, da man von einer Beteiligung des Opioidssystems bei der Entstehung des RLS ausgeht und einen positiven Effekt bei Gabe von Opioiden beobachtet (Hening W et al. 1999). Studien haben gezeigt, dass 
Naloxon zwar zu einer Verschlechterung der Symptome bei RLS-Patienten führt, die mit Opioiden vorbehandelt sind (Hening et al. 1986). Bei unbehandelten Patienten, denen Naloxon verabreicht wird, kommt es allerdings zu keiner Verschlechterung der Symptomatik (Winkelmann et al. 2001). Auch wird die Wirkung der dopaminergen Therapie nicht negativ durch Naloxon beeinflusst (Tribl et al. 2005).

Obwohl sich in vorliegender Studie kein Patient befand, bei dem durch die Anästhesie ein RLS ausgelöst oder verstärkt wurde, kann nicht völlig ausgeschlossen werden, dass trotz dieses Ergebnisses ein kausaler Zusammenhang besteht. Betrachtet man das gesamte Kollektiv von 398 Patienten, kann man bei einem 99\% Konfidenzintervall davon ausgehen, dass das Risiko, durch eine Narkose ein RLS neu zu entwickeln bzw. zu verschlechtern, kleiner als 1,3\% ist. Dieser Wert gibt bereits das Maximum der Wahrscheinlichkeit an. Der tatsächliche Wert kann durchaus noch kleiner oder gleich null sein. Eine Betrachtung des gesamten Kollektivs ist gerechtfertigt, da nicht ausser Acht gelassen werden darf, dass nicht nur die Anästhesie, sondern auch andere Faktoren wie die durch die Operation ausgelöste Entzündung (Nemeth und Ganz 2006), die Immobilisation (Michaud et al. 2002a) oder der postoperativ auftretende Eisenmangel, eine Rolle bei der Entstehung des RLS spielen.

Betrachtet man nun gesondert die Patienten mit SPA, von denen ebenfalls keiner postoperativ neu aufgetretene Symptome eines RLS entwickelte, liegt die Wahrscheinlichkeit, dass ein RLS doch auftreten könte, mit 99\% Wahrscheinlichkeit zwischen null und 4,0\%. Dieser theoretische Wert liegt allerdings noch deutlich unter dem Wert von 8\% aus der Studie von Högl et al. (2002).

Nach Auswertung der Ergebnisse dieser Studie kommen wir zu der Schlussfolgerung, dass ein kausaler Zusammenhang zwischen dem Auftreten des RLS und einer Anästhesie bzw. SPA sehr unwahrscheinlich ist. Hauptursächlich für 
die stark voneinander abweichenden Ergebnisse scheinen Unterschiede im methodischen Vorgehen der Studien zu sein.

Es wäre wünschenswert, wenn die Ergebnisse dieser Studie in die Empfehlungen und Leitlinien für Anästhesisten, sowie der Deutschen Restless Legs Vereinigung (RLS e.V.) aufgenommen würden, um Patienten, die unter einem RLS leiden, Bedenken vor einem operativen Eingriff, der ggf. mit einer SPA durchgeführt werden könnte, zu nehmen, und gesunde Patienten nicht unnötig zu beunruhigen. 


\section{Zusammenfassung}

Das Restless-Legs-Syndrom ist eine relativ häufige neurologische Erkrankung, der wahrscheinlich eine Störung des dopaminergen Neurotransmittersystems zugrunde liegt. Von der idiopathischen, primären Form unterscheidet man eine sekundäre Form, die durch identifizierbare auslösende Faktoren verursacht wird, zu denen auch anästhesierelevante Pharmaka gehören. Es wurde auch ein kausaler Zusammenhang mit der Spinalanästhesie postuliert, die in ca. $8 \%$ der Fälle ein Restless Legs Syndrom auslösen soll.

Dieser mögliche Zusammenhang wurde in einer prospektiven, kontrollierten longitudinalen Studie untersucht, in der das perioperative Auftreten des RestlessLegs-Syndrom bei Patienten mit chirurgischen Operationen in Spinalanästhesie anhand der Kriterien der IRLS ermittelt wurde. Als Kontrollgruppen dienten Patienten mit Eingriffen in Allgemeinanästhesie bzw. peripherer Regionalanästhesie.

Präoperativ, sowie eine und vier Wochen postoperativ wurden im persönlichen Interview anhand eines standardisierten Fragebogens die diagnostischen Kriterien der International Restless Legs Society abgefragt. Bei Anhaltspunkten für das Vorliegen eines RLS schloss sich eine genauere neurologische Abklärung an.

Es wurden insgesamt 398 Patienten untersucht: 147 mit Spinalanästhesie, 212 mit Allgemeinanästhesie und 39 mit peripherer Regionalanästhesie. Die präoperative RLS-Prävalenz unseres Kollektivs lag mit 7,8\% im üblichen Bereich deutscher Studienpopulationen; Frauen und Ältere waren häufiger betroffen. Bei keinem Patienten wurde ein de-novo-Auftreten von RLS beobachtet, und kein Patient mit vorbestehendem RLS berichtete von einer postoperativen Symptomverschlechterung. 
Die Daten legen die Schlussfolgerung nahe, dass kein kausaler Zusammenhang zwischen Spinalanästhesie und dem Auftreten eines Restless-Legs-Syndroms besteht. Die aus den Daten errechnete Wahrscheinlichkeit, dass ein Restless-LegsSyndrom nach Spinalanästhesie auftreten wird, liegt mit 99\%iger Sicherheit zwischen $0 \%$ und maximal $4,0 \%$. 


\section{Literaturverzeichnis}

Akpinar S (1987): Restless Legs Syndrome Treatment with Dopaminergic Drugs. Clin Neuropharmacol 10 (1): 69-79.

Allen RP, Earley CJ (1996): Augmentation of the restless legs syndrome with carbidopa/levodopa. Sleep 19 (3): 205-213.

Allen RP, Earley CJ (2001): Restless Legs Syndrome: a review of clinical and pathophysiological features. J Clin Neurophysiol 18: 128-147.

Allen RP, Earley CJ (2007): The role of iron in restless legs syndrome. Mov Disord 22 Suppl 18: S440-448.

Allen RP, Picchietti D, Hening WA, Trenkwalder C, Walters AS, Montplaisir J, the participants in the Restless Legs Syndrome Diagnosis and Epidemiology workshop at the National Institutes of Health in collaboration with members of the International Restless Legs Syndrome Study Group (2003): Restless legs syndrome: diagnostic criteria, special considerations, and epidemiology - A report from the restless legs syndrome diagnosis and epidemiology workshop at the National Institutes of Health. Sleep Med 4: 101-119.

Auger C, Montplaisir J, Duquette P (2005): Increased frequency of restless legs syndrome in a French- Canadian population with multiple sclerosis. Neurology 65 (10): 1652-1653.

Bara-Jimenez W, Aksu M, Graham B, Sato S, Hallett M (2000): Periodic limb movements in sleep: state-dependent excitability of the spinal flexor reflex. Neurology 54 (8): 1609-1616.

Barnes TR, Braude WM, Hill DJ (1982): Acute akathesia after oral droperidol and metoclopramide preoperative medication. Lancet 1982, 2 (8288): 48-49.

Bassetti C, Mauerhofer D, Gugger M, Mathis J, Hess CW (2001): Restless legs Syndrome: a clinical study of 55 patients. Eur Neurol 45: 67-74.

Benes H (2006): Transdermal Lisuride: First Efficacy and Tolerability Study in Patients with severe Restless Legs Syndrome. Sleep Med 7: 31-35. 
Benes H, Kurella B, Kummer J, Kazenwadel J, Selzer R, Kohnen R (1999): Rapid onset of action of levodopa in restless legs syndrome: a double- blind, randomized, multicenter, crossover trial. Sleep 22: 1073-1081.

Berger K, von Eckardstein A, Trenkwalder C, Rothdach A, Junker R, Weiland SK (2002): Iron metabolism and the risk of restless legs syndrome in an elderly general population - the MEMO Study. J Neurol 249 (9): 1195-1199.

Berger K, Luedemann J, Trenkwalder C, John U, Kessler C (2004): Sex and the Risk of Restless Legs Syndrome in the General Population. Arch Intern Med 164 (2): 196202.

Bier A (1899): Versuche über die Cocainisierung des Rückenmarks. Dtsch Z Chir 51: 361-368.

Boghen D (1980): Successful treatement of restless legs with clonazepam. Ann Neurol 8 (3): 341.

Brodeur C, Montplaisir J, Godbout R, Marinier R (1988): Treatment of restless legs syndrome and periodic movements during sleep with L-dopa: a double-blind, controlled study. Neurology 38 (12): 1845-1848.

Bucher SF, Trenkwalder C, Oertel WH (1996): Reflex studies and MRI in the restless legs syndrome. Acta Neurol Scand 94 (2): 145-150.

Bucher SF, Seelos KC, Oertel WH, Reiser M, Trenkwalder C (1997): Cerebral generators involved in the pathogenesis of the restless legs syndrome. Ann Neurol 41 (5): 639-645.

Buysse DJ, Reynolds CF 3rd, Monk TH, Berman SR, Kupfer DJ (1989): The Pittsburgh Sleep Quality Index: a new instrument for psychiatric practice and research. Psychiatry Res 28 (2): 193-213.

Cho YW, Shin WC, Yun CH, Hong SB, Kim JH, Allen RP, Earley CJ (2008): Epidemiology of restless legs syndrome in Korean adults. Sleep 31 (2): 219-223.

Cohrs S, Rodenbeck A, Hornyak M, Kunz D (2008): Restless-legs-Syndrom, periodische Gliedmaßenbewegungen im Schlaf und Psychopharmakologie. Nervenarzt 79: 1263-1272. 
Collado-Seidel V, Kohnen R, Samtleben W, Hillebrand GF, Oertel WH,Trenkwalder C (1998): Clinical and Biochemical Findings in Uremic Patients With and Without Restless legs Syndrome. Am J Kidney Dis 31 (2): 324-328.

Conti CF, de Oliveira MM, Valbuza JS, Prado LB, de Carvalho LB, do Prado GF (2008): Anticonvulsants to treat idiopathic restless legs syndrome: systematic review. Arg Neuropsiquiatr 66 (2B): 431-435.

Corning JL (1885): Spinal anaesthesia and local medication of the cord. $N Y$ State Med J 42: 483.

Drake ME (1988): Restless legs with antiepileptic drug therapy. Clin Neurol Neurosurg 90 (2): 151-154.

Duggal HS, Mendhekar DN (2007): Clozapine-associated restless legs syndrome. J Clin Psychopharmacol 27 (1): 89-90.

Earley CJ, Yaffee JB, Allen RP (1998): Randomized, double-blind, placebo-controlled trial of pergolide in restless legs syndrome. Neurology 51: 1599-1602.

Earley CJ, Allen RP, Beard JL, Connor JR (2002): Insight into the pathophysiology of restless legs syndrome. J Neurosci Res 62: 632-628.

Earley CJ, Heckler D, Allen RP (2005): Repeated IV doses of iron provides effective supplemental treatment of restless legs syndrome. Sleep Med 6 (4): $301-305$.

Eisensehr I, Wetter TC, Linke R, Noachtar S, von Lindeiner H, Gildehaus FJ, Trenkwalder C, Tatsch K (2001): Normal IPT and IBZM SPECT in drug-naive and levodopa-treated idiopathic restless legs syndrome. Neurology 57: 1307-1309.

Eisensehr I, Ehrenberg BL, Rogge Solti S, Noachtar S (2004): Treatement of idiopathic restless legs syndrome (RLS) with slow-release valproic acid compared with slow-release levodopa/benserazid. J Neurol 251 (5): 579-583.

Ekbom KA (1945): Restless legs syndrome. Acta Med Scand 158 (Suppl.): 4-122.

Ekbom KA (1960): Restless legs syndrome. Neurology 10: 868-873.

Entezari-Taher M, Singleton JR, Jones CR, Meekins G, Petajan JH, Smith AG (1999): Changes in excitability of motor cortical circuitry in primary restless legs syndrome. Neurology 53 (6): 1201-1205. 
Foster PN, Stickle BR, Laurence AS (1996): Akathisia following low-dose droperidol for antiemesis in day-case patients. Anaesthesia 51 (5): 491-494.

Furnham A (1986): Response bias, social desirability and dissimulation. Person Individ Diff 7: 385-400.

Garcia-Borreguero D, Larrosa O, de la Llave Y, Verger K, Masramon X, Hernandez $G$ (2002): Treatment of restless legs syndrome with gabapentin: a double-blind, cross-over study. Neurology 59 (10): 1573-1579.

Gemignani F, Marbini A (2002): Restless legs syndrome and peripheral neuropathy. Neurol Neurosurg Psychiatry 72 (4): 555.

Gemignani F, Brindani F (2007): Restless legs syndrome associated with peripheral neuropathy. Eur J Neurol 14 (7): e9-e10.

Gómez-Esteban JC, Zarranz JJ, Tijero B, Barcena J, Rouco I, Lezano E, Lachen MC, Jauregui A, Ugarte A (2007): Restless legs syndrome in Parkinson's disease. Mov Disord 22 (13): 1912-1916.

Goodman JD, Brodie C, Ayida GA (1988): Restless legs syndrome in pregnancy. BMJ 297 (6656): 1101-1102.

Happe S, Sauter C, Klösch G, Saletu B, Zeitlhofer J (2003): Gabapentin versus ropinirole in the treatement of idiopathic restless legs syndrome. Neuropsychobiology 48 (2): 82-86.

Hening W (2004): The clinical neurophysiology of the restless legs syndrome and periodic limb movements. Part I: diagnosis, assessment and characterization. Clin Neurophysiol 115 (9): 1965-1974.

Hening W, Allen R, Earley CJ, Kushda C, Picchietti D, Silber M (1999): The treatment of restless legs syndrome and periodic limb movement disorder. Sleep 22: 970-998.

Hening WA, Walters AS, Kavey N, Gidro-Frank S, Côté L, Fahn S (1986): Dyskinesias while awake and periodic movements in sleep in restless legs syndrome: Treatment with opioids. Neurology 36: 1363-1364.

Hening WA, Walters AS, Wagner M, Rosen R, Chen V, Kim S, Shah M, Thai O (1999): Circadian rhythm of motor restlessness and sensory symptoms in the idiopathic restless legs syndrome. Sleep 22: 901-912. 
Högl B, Frauscher B, Seppi K, Ulmer H, Poewe W (2002): Transient restless legs syndrome after spinal anesthesia-A prospective study. Neurology 59: 1705-1707.

Högl B, Kiechl S, Willeit J, Saletu M, Frauscher B, Seppi K, Müller J, Rungger G, Gasperi A, Wenning G, Poewe W (2005): Restless legs syndrome: a communitybased study of prevalence, severity, and risk factors. Neurology 64 (11): 1920-1924.

Hornyak M, Feige B, Riemann D, Voderholzer U (2006): Periodic leg movements in sleep and periodic limb movement disorder: prevalence, clinical significance and treatment. Sleep Med Rev 10 (3): 169-177.

Hornyak M, Feige B, Vorderholzer U, Philipsen A, Riemann D (2007): Polysomnography findings in patients with restless legs syndrome and in healthy controls: a comparative observational study. Sleep 30 (7): 861-865.

Jiménez-Jiménez FJ, García-Ruiz PJ, Molina JA (1997): Drug-induced movement disorders. Drug Saf 16 (3):180-204.

Johns MW (1991): A new method for measuring daytime sleepiness: the Epworth sleepiness scale. Sleep 14 (6): 540-545.

Karroum E, Konofal E, Arnulf I (2008): Restless- legs syndrome. Rev Neurol (Paris) 164 (8-9): 701-721.

Kavanagh D, Siddiqui S, Geddes CC (2004): Restless legs syndrome in patients on dialysis. Am J Kidney Dis 43 (5): 763-771.

Kvernmo T, Houben J, Sylte I (2008): Receptor-binding and pharmacokinetic properties of dopaminergic agonists. Curr Top Med Chem 8 (12): 1049-1067.

Lavigne GJ, Montplaisir JY (1994): Restless Legs Syndrome and Sleep Bruxism: Prevalence and Association Among Canadians. Sleep 17 (8): 739-743.

Lazzarini A, Walters AS, Hickey K, Coccagna G, Lugaresi E, Ehrenberg BL, Picchietti DL, Brin MF, Stenroos ES, Verrico T, Johnson WG (1999): Studies of penetrance and anticipation in five autosomal-dominant restless legs syndrome pedigrees. Mov Disord 14 (1): 111-116.

Lugaresi E, Cirignotta F, Coccagna G, Montagna P (1986): Nocturnal myoclonus and restless legs syndrome. Adv Neurol 43: 295-307. 
Lundvall O, Abom PE, Holm R (1983): Carbamazepine in restless legs. A controlled pilot study. Eur J Clin Pharmacol 25 (3): 323-324.

Lutz EG (1978): Restless legs, anxiety and caffeinism. J Clin Psychiatry 39 (9): 693698.

Manconi M, Govoni V, De Vito A, Economou NT, Cesnik E, Casetta I, Mollica G, Ferini-Strambi L, Garnieri E (2004): Restless legs syndrome and pregnancy. Neurology 63 (6): 1065-1069.

Markkula J, Lauerma H (1997): Mianserin and restless legs. Int Clin Psychopharmacol 12 (1): 53-58.

Meinck HM, Möhlenhof O, Kettler D (1980): Neurophysiological effects of etomidate, a new short-acting hypnotic. Electroencephalogr. Clin Neurophysiol 50: 515-522.

Merlino G, Fratticci L, Valente M, Del Giudice A, Noacco C, Dolso P, Cancelli I, Scalise A, Gigli GL (2007): Association of Restless Legs Syndrome in Type 2 Diabetes: A Case-Control Study. Sleep 30 (7): 866-871.

Michaud M, Chabli A, Lavigne G, Montplaisir J (2000): Arm restlessness in patients with restless legs syndrome. Mov Disord 15: 289-293.

Michaud M, Lavigne G, Desautels, Poirier G, Montplaisir J (2002a): Effects of immobility on sensory and motor symptoms of restless legs syndrome. Mov Disord 17: 112-115.

Michaud M, Soucy JP, Chabli A, Lavigne G, Montplaisir J (2002b): SPECT imaging of striatal pre- and postsynaptic dopaminergic status in restless legs syndrome with periodic leg movements in sleep. J Neurol 249 (2): 164-170.

Mizuno S, Mihara T, Miyaoka T, Inagaki T, Horiguchi J (2005): CSF iron, ferritin and transferrin levels in restless legs syndrome. Sleep Res 14 (1): 43-47.

Modica PA, Tempelhoff R, White PF (1990): Pro- and anticonvulsant effects of anesthetics (Part I, II). Anesth Analg 70: 303-315,433-444.

Molnar MZ, Novak M, Ambrus C, Szeifert L, Kovacs A, Pap J, Remport A, Mucsi I (2005): Restless Legs Syndrome in patients after renal transplantation. Am J Kidney Dis 45 (2): 388-396. 
Montplaisir J, Godbout R, Poirier G, Bédard M (1986): Restless Legs Syndrom and Periodic Movements in Sleep: Physiopathology and Treatment with L-Dopa. Clin Neuropharmacol 9 (5): 456-463

Montplaisir J, Boucher S, Poirier G, Lavigne G, Lapierre O, Lésperance P (1997): Clinical, polysomnographic, and genetic characteristics of restless legs syndrome: a study of 133 patients diagnosed with new standard criteria. Mov Disord 12 (1): 61-65.

Montplaisir J, Boucher S, Nicolas A, Lesperance P, Gosselin A, Rompré P, Lavigne $G$ (1998): Immobilization tests and periodic leg movements in sleep for the diagnosis of restless legs syndrome. Mov Disord 13 (2): 324-329.

Montplaisir J, Nicolas A, Denesle R, Gomez-Mancilla B (1999): Restless legs syndrome improved by pramipexole: a double-blind randomized trial. Neurology 52: 938-943.

Montplaisir J, Denesle R, Petit D (2000): Pramipexole in the treatment of restless legs syndrome: a follow-up study. Eur J Neurol 7 Suppl 1: 27-31.

Nemeth E, Ganz T (2006): Regulation of iron matabolism by hepcidin. Annu Rev Nutr 26: 323-342.

Nichols DA, Allen RP, Grauke JH, Brown JB, Rice ML, Hyde PR, Dement WC,

Kushida CA (2003): Restless Legs Syndrome Symptoms in Primary Care- A Prevalence Study. Arch Intern Med 163: 2323-2329.

Oertel WH, Benes H, Bodenschatz R, Peglau I, Warmuth R, Happe S, Geisler P, Cassel W, Leroux M, Kohnen R, Stiansky-Kolster K (2006): Efficacy of cabergoline in restless legs syndrome: a placebo-controlled study with polysomnography (CATOR). Neurology 67 (6): 1040-1046.

O'Keeffe ST, Gavin K, Lavan JN (1994): Iron status and restless legs syndrome in the elderly. Age Ageing 23 (3): 200-203.

Ondo W, Tan EK, Mansoor J (2000): Rheumatologic serologies in secondary restless legs syndrome. Mov Disord 15 (2): 312-323.

Ondo WG, Dat Vuong KD, Jankovic J (2002): Exploring the relationship betweeen Parkinson disease and restless legs syndrome. Arch Neurol 59: 421-424. 
Patrick LR (2007): Restless legs syndrome: pathophysiology and the role of iron and folate. Altern Med Rev 12 (2): 101-112.

Paulson GW (2000): Restless legs syndrome. How to provide symptom relief with drug and nondrug therapies. Geriatrics 55 (4): 35-38, 43-44, 47-48.

Paulus W, Trenkwalder C (2006): Less is more: pathophysiology of dopaminergictherapy-related augmentation in restless legs syndrome. Lancet Neurol 5 (10): 878886.

Paulus W, Dowling P, Rijisman R, Stiasny-Kolster K, Trenkwalder C (2007): Update of the pathophysiology of the restless legs syndrome. Mov Disord 22 Suppl. 18: S431-S439.

Phillips B, Young T, Finn L, Asher K, Hening WA, Purvis C (2000): Epidemiology of restless legs symtoms in adults. Arch Intern Med 160 (14):2137-2141.

Picchietti DL, Walters AS (1996): Restless legs syndrome and periodic limb movement disorder in children and adolescents. Comorbidity with attention-deficithyperactivity disorder. Neurol Clin 5: 729-740.

Picchietti MA, Picchietti DL (2008): Restless legs syndrome and periodic limb movement disorder in children and adolescents. Semin Pediatr Neurol 15 (2): 91-99.

Provini F, Vetrugno R, Meletti S, Plazzi G, Solieri L, Lugaresi E, Coccagna G, Montagna $P$ (2001): Motor pattern of periodic limb movements during sleep. Neurology 57 (2): 300-304.

Quincke HI (1891): Die Lumbalpunktion des Hydrocephalus. Berl Med Wochenschr 28: 929-33.

Radloff LS (1977): The CES-D Scale: A Self-Report Depression Scale for Research in the General Population. Appl Psychol Meas 1: 385-401

Rangarajan S, Rangarajan S, D'Souza GA (2007): Restless legs syndrome in an Indian urban population. Sleep Med 9 (1): 88-93.

Reddy RV, Moorthy SS, Dierdorf SF, Deitch RD Jr, Link L (1993): Excitatory effects and electroencephalographic correlation of etomidate, thiopental, methohexital and propofol. Anesth Analg 77(5): 1008-1011. 
Rothdach AJ, Trenkwalder C, Haberstock J, Keil U, Berger K (2000): Prevalence and risk factors of RLS in an elderly population: The MEMO Study. Neurology 54: 10641068.

Ruottinen HM, Partinen M, Hublin C, Bergman J, Haaparanta M, Solin O, Rinne JO (2000): An FDOPA PET study in patients with periodic limb movement disorder and restless legs syndrome. Neurology 54 (2): 502-504.

Rüsch D, Eberhart L, Biedler A, Dethling J, Apfel CC (2005): Prospective application of a simplified risk score to prevent postoperative nausea and vomiting. Can $J$ Anaest 52: 478-484.

Saletu M, Anderer P, Saletu-Zyhlarz G, Prause W, Semler B, Zoghlami A, Gruber G, Hauer C, Saletu B (2001): Restless legs syndrome (RLS) and periodic limb movement disorder (PLMD): acute placebo-controlled sleep laboratory studies with clonazepam. Eur Neuropsychopharmacol 11 (2): 153-161.

Salih AM, Gray RE, Mills KR, Webley M (1994): A clinical, serological and neurophysiological study of restless legs syndrome in rheumatoid arthritis. $\mathrm{Br} J$ Rheumatol 33 (1): 60-63.

Santos A, Datta S (1984): Prophylactic use of droperidol for control of nausea and vomiting during spinal anesthesia for cesarean section. Anesth Analg 63: 85-87.

Schober T, Wenzel K, Feichtinger M, Schwingenschuh P, Strebel A, Krausz G, Pfurtscheller G (2004): Restless legs syndrome: changes of induced electroencephalographic beta oscillations-an ERD/ERS study. Sleep 27 (1): 147-150.

Siddiqui S, Kavanagh D, Traynor J, Mak M, Deighan C, Geddes C (2005): Risk factors for restless legs syndrome in dialysis patients. Nephron Clin Pract 101 (3): 155-160.

Staedt J, Stoppe G, Kögler A, Riemann H, Hajak G, Munz DL, Emrich D, Rüther E (1995): Nocturnal myoclonus syndrome (periodic movements in sleep) related to central dopamine D2-receptor alteration. Eur Arch Psychiatry Clin Neurosci 245 (1): 8-10.

Staedt J, Hunerjager H, Ruther E, Stoppe G (1998): Pergolide: treatment of choice in restless legs syndrome (RLS) and nocturnal myoclonus syndrome (NMS). Longterm follow up on pergolide. J Neural Transm 105: 265-268. 
Stein DJ, Birnbach DJ, Danzer BI, Kuroda MM, Grunebaum A, Thys DM (1997): Acupressure versus intravenous metoclopramide to prevent nausea and vomiting during spinal anesthesia for cesarean section. Anesth Analg 84: 342-345.

Stiasny K, Wetter TC, Trenkwalder C, Oertel WH (2000): Restless legs syndrome and its treatment by dopamine agonists. Parkinsonism Relat Disord 7 (1): 21-25.

Stiasny K, Haeske H, Tergau F, Müller HH, Braune HJ, Oertel WH (2003): Cortical silent period is shortened in restless legs syndrome independently from circadian rhythm. Suppl Clin Neurophysiol 56: 381-389.

Stiasny-Kolster K, Kohnen R, Moller JC, Trenkwalder C, Oertel W (2006): Validation of the "L-DOPA test" for diagnosis of restless legs syndrome. Mov Disord 10: 634642.

Sun ER, Chen CA, Ho G, Earley CJ, Allen RP (1998): Iron and the restless legs syndrome. Sleep 21 (4): 371-377.

Tacconi P, Manca D, Tamburini G, Ferrigno P, Cossu G, Cannas A, Giagheddu M (2004): Electroneurography index based on nerve conduction study data: method and findings in control subjects. Muscle Nerve 29 (1): 89-96.

Terao T, Terao M, Yoshimura R, Abe K (1991): Restless legs syndrome induced by lithium. Biol Psychiatry 30 (11): 1167-1170.

Tergau F, Wischer S, Paulus W (1999): Motor system excitability in patients with restless legs syndrome. Neurology 52 (5): 1060-1063.

Trenkwalder C (2006): The weight of evidence for ropinirole in restless legs syndrome. Eur J Neurol 13 Suppl 3: 21-30.

Trenkwalder C: Restless Legs Syndrom- Leitfaden für Anästhesisten. (URL www.slb.de/Bild_gif/RLS\%20f\%FCr\%20An\%E4sthesisten.pdf. zuletzt aufgerufen am 7.01.2009)

Trenkwalder C, Bucher SF, Oertel WH, Proeckl D, Plendl H, Paulus W (1993):

Bereitschaftspotential in idiopathic and symptomatic restless legs syndrome. Electroencephalogr Clin Neurophysiol 89(2):95-103. 
Trenkwalder C, Stiasny K, Pollmächer T, Wetter T, Schwarz J, Kohnen R, Kazenwadel J, Krüger HP, Ramm S, Künzel M, et al. (1995): L-dopa therapy of uremic and idiopathic restless legs syndrome: a double-blind, crossover trial. Sleep 18: $681-688$.

Trenkwalder C, Seidel VC, Gasser T, Oertel WH (1996): Clinical symptoms and possible anticipation in a large kindred of familial restless legs syndrome. Mov Disord 11 (4): 389-394.

Trenkwalder C, Hening WA,Walters AS, Campbell SS, Rahman K, Chokroverty S (1999a): Circadian rhythm of periodic limb movements and sensory symptoms of restless legs syndrome. Mov Disord 14: 102-110.

Trenkwalder C, Walters AS, Hening WA, Chokroverty S, Antonini A, Dhawan V, Eidelberg D (1999b): Positron emission tomographic studies in restless legs syndrome. Mov Disord 14(1): 141-145.

Trenkwalder C, Hundemer HP, Lledo A, Swieca J, Polo O, Wetter TC, Ferini-Strambi L, de Groen H, Quail D, Brandenburg U, PEARLS Study Group (2004): Efficacy of pergolide in treatment of restless legs syndrome: the PEARLS Study. Neurology 62 (8): 1391-1397.

Trenkwalder C, Högl B, Benes H, Kohnen R (2008a): Augmentation in restless legs syndrome is associated with low ferritin. Sleep Med 9 (5): 572-574.

Trenkwalder C, Hening WA, Montagna P, Oertel WH, Allen RP, Walters AS, Costa J, Stiasny-Kolster K, Sampaio C (2008b): Treatment of restless legs syndrome: An evidence-based review and implications for clinical practice. Mov Disord DOI 23 (16): 2267-2302.

Tribl GG, Asenbaum S, Klösch G, Mayer K, Bonelli RM, Auff E, Zeitlhofer J, Happe S (2002): Normal IPT and IBZM SPECT in drug-naive and levodopa-treated idiopathic restless legs syndrome. Neurology 59 (4): 649-650.

Tribl GG, Sycha T, Kotzailias N, Zeitlhofer J, Auff E (2005): Apomorphine in idiopathic restless legs syndrome: an exploratory study. J Neurol Neurosurg Psychiatry 76: 181-185.

Tunç T, Karadag YS, Dogulu F, Inan LE (2007): Predisposing factors of restless legs syndrome in pregnancy. Mov Disord 22 (5): 627-631. 
Turjanski N, Lees AJ, Brooks DJ (1999): Striatal dopaminergic function in restless legs syndrome: 18F-dopa and 11C-raclopride PET studies. Neurology 52 (5): 932937.

Van Keulen SG, Burton JH (2003): Myoclonus associated with etomidate for ED procedural sedation and analgesia. Am J Emerg Med 21 (7): 556-558.

Voss LJ, Sleigh JW, Barnard JP, Kirsch HE (2008): The howling cortex: seizures and general anesthetic drugs. Anesth Analg 107:1689-1703.

Walters AS (1995): Toward a better definition of the restless legs syndrome. The International Restless Legs Syndrome Study Group. Mov Disord 10 (5): 634-642.

Walters AS (2002): Review of receptor agonist and antagonist studies relevant to the opiate system in restless legs syndrome. Sleep Med 3(4): 301-4.

Walters AS, Hening WA, Kavey N, Chokroverty S, Gidro-Frank S (1988): A doubleblind randomized crossover trial of bromocriptine and placebo in restless legs syndrome. Ann Neurol 24: 455-458.

Walters AS, Hening W, Rubinstein M, Chokroverty S (1991): A clinical and polysomnographic comparison of neuroleptic-induced akathisia and the idiopathic restless legs syndrome. Sleep 14 (4): 339-345.

Walters AS, Wagner ML, Hening WA, Grasing K, Mills R, Chokroverty S, Kavey N (1993): Successful treatment of the idiopathic restless legs syndrome in a randomized double-blind trial of oxycodone versus placebo. Sleep 16 (4): 327-332.

Walters AS, Hickey K, Maltzman J, Verrico T, Joseph D, Hening W, Wilson V, Chokroverty S (1996): A questionnaire study of 138 patients with restless legs syndrome: the 'Night-Walkers' survey. Neurology 46: 92-95.

Walters AS, Winkelmann J, Trenkwalder C, Fry JM, Kataria V, Wagner M, Sharma R, Hening W, Li L (2001): Long-term follow-up on restless legs syndrome patients treated with opioids. Mov Disord 16 (6): 1105-1109.

Walters AS, LeBroq C, Dhar A, Hening W, Rosen R, Allen RP, Trenkwalder C; International Restless Legs Syndrome Study Group (2003): Validation of the International Restless Legs Syndrome Study Group rating scale for restless legs syndrome. Sleep Med 4: 121-132. 
Ward NG (1989): Akathisia associated with droperidol during epidural anesthesia. Anesthesiology 71 (5): 786-787.

Wetter TC, Stiasny K, Winkelmann J, Buhlinger A, Brandenburg U, Penzel T, Medori R, Rubin M, Oertel WH, Trenkwalder C (1999): A randomized controlled study of pergolide in patients with restless legs syndrome. Neurology 52: 944-950.

Wetter TC, Brunner J, Bronisch T (2002): Restless legs syndrome probably induced by risperidone treatment. Pharmacopsychiatry 35 (3): 109-111.

Willis T: The London Practice of Physick. Basset \& Crooke, London, 1685.

Winkelmann J, Ferini-Strambi L (2006): Genetics of restless legs syndrome. Sleep Med Rev 10 (3): 179-183.

Winkelmann J, Wetter TC, Collado-Seidel V, Gasser T, Dichgans M, Yassouridis A, Trenkwalder C (2000): Clinical characteristics and frequency of the hereditary restless legs syndrome in a population of 300 patients. Slee. 23: 597-602.

Winkelmann J, Schadrack J, Wetter TC, Zieglgänsberger W, Trenkwalder C (2001): Opioid and dopamine antagonist drug challenges in untreated restless legs syndrome patients. Sleep Med 2 (1): 57-61.

Winkelmann J, Prager M, Lieb R, Pfister H, Spiegel B, Wittchen HU, Holsboer F, Trenkwalder C, Strohle A (2005): „Anxietas tibiarum“. Depression and anxiety disorders in patients with restless legs syndrome. J Neurol 252 (1): 67-71.

Winkelmann J, Lichtner P, Pütz B, Trenkwalder C, Hauk S, Meitinger T, Strom T, Muller-Myhsok B (2006): Evidence for further genetic locus heterogeneity and confirmation of RLS-1 in restless legs syndrome. Mov Disord 21 (1):28-33.

Winkelmann J, Schormair B, Lichtner P, Ripke S, Xiong L, Jalilzadeh S, Fulda S, Pütz B, Eckstein G, Hauke S, Trenkwalder C, Zimprich A, Stiasny-Kolster K, Oertel W, Bachmann CG, Paulus W, Peglau I, Eisensehr I, Monplaisir J, Turecki G, Rouleau G, Gieger C, Illig T, Wichmann HE, Holsboer F, Müller-Myhsok B, Meitinger T (2007): Genome-wide association study of restless legs syndrome identifies common variants in three genomic regions. Nat Genet 39 (8): 1000-1006.

Wittmaack T: Pathologie and Therapie des Sensibilitats-Neurosen. Lehrbuch der Nervenkrankheiten, Teil 1: Pathologie and Therapie der sensiblen Neurosen. E.Schafer, Leipzig, 1861. 
Zucconi M, Ferini-Strambi L (2004): Epidemiology and clinical findings of restless legs syndrome. Sleep Med 5: 293-299.

Zucconi M, Coccagna G, Petronelli R, Gerardi R, Mondini S, Cirignotta F (1989): Nocturnal myoclonus in restless legs syndrome effect of carbamazepin treatment. Funct Neurol 4 (3): 263-271.

Zucconi M, Ferri R, Allen RP, Baier PC, Bruni O, Chokroverty S, Ferini-Strambi L, Fulda S, Garcia-Borreguero D, Hening WA, Hirschkowitz M, Högl B, Hornyak M, King M, Montagna P, Parrino L, Plazzi G, Terzano MG (2006): The official World Association of Sleep Medicine (WASM) standards for recording and scoring periodic leg movements in sleep (PLMS) and wakefulness (PLMW) developed in collaboration with a task force from the International Restless Legs Syndrome Study Group (IRLSSG). Sleep Med 7 (2): 175-183. 


\section{Tabellenverzeichnis}

Tabelle 1: Seite 11

Tabelle 2: Seite 11

Tabelle 3: Seite 24

Tabelle 4: Seite 41

Tabelle 5: Seite 43

Tabelle 6: Seite 44 


\section{Anhang: Fragebogen}

\section{Laufende Nr:}

Patient in Studie eingeschlossen am: $\quad$. . . (MM..TT.JJ)

Datum der Befragung: $\quad$. (MM..TT.JJ)

Name:

Adresse:

Telefon:

Grund für die stationäre Aufnahme:

Körpergewicht: $\quad \mathrm{kg}$

Körpergröße: $\quad \mathrm{cm}$

Geb. Datum: . (MM..TT.JJ)

Geschlecht: M / W; (bei Frauen ggf.) Anzahl der Kinder: $\quad$ Schwanger? J / N Rauchen: J / N

Aktuellste Laborwerte (soweit vorliegend):

$\mathrm{Hb}$ :. $\mathrm{g} \mathrm{dl}^{-1}$

$\mathrm{T}_{3}$ : $\mathrm{pg} / \mathrm{ml}$

(soweit

vorhanden)

MCV $\mathrm{fl}$

$\mathrm{T}_{4}:$ $\mathrm{mg} / \mathrm{dl}$ (soweit

vorhanden)

$\mathrm{MCH}$ : pg

TSH: $\mu \mathrm{U} / \mathrm{ml}$ (soweit

vorhanden)

Kreatinin: $\mathrm{mg} \mathrm{dl}^{-1}$

Eisen: $\mu \mathrm{mol} / \mathrm{I}$ (soweit vorhanden)

Haptoglobin:. g/l (soweit vorhanden) 
Transferrin: $\mathrm{mg} / \mathrm{dl}$ (soweit vorhanden)

Behandlung: operativ J / N konservativ: J / N Lumbalpunktion? ? J / N Vorerkrankungen:

Vormedikation:

OP-relevante Erkrankung:

Operation bzw. sonstige Therapie:

OP-Datum: . . (MM.TT.JJ)OP-Dauer: Minuten

Mobilisation ab Tag: ; bzw. Bettruhe: Tage

Verweildauer im Krankenhaus: Tage; Normale Nahrungsaufnahme ab Tag:

Medikamente:

Bemerkungen:

Anästhesieverfahren (Verfahren ankreuzen - Mehrfachnennung möglich)
Allgemeinanästhesie
Spinalanästhesie (Nadel

Periduralanästhesie

periphere Regionalanästhesie Lokalanästhesie_ LA mit Sedierung

\section{verwendete Anästhetika (Substanzen und Dosierungen genau angeben)}

Substanzgruppe? welche Substanzen? Dosis

Benzodiazepine: ......................................... mg 


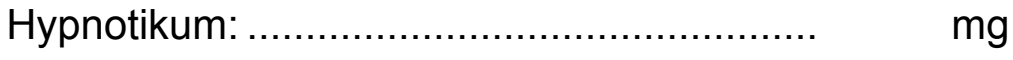

Opiate: ...................................... $\mathrm{mg}$

Ketamin (razemisch? S-(+)?): ................................. mg

volatiles Anästhetikum: ........................................ MAC-hours

a-2 Agonisten (z.B. Clonidin): .................................... mg

Lokalanästhetikum: ...................................... $\mathrm{ml}$

(bei SPA und PDA)

Akrinor: ...................................... $\mathrm{ml}$

Katecholamininfusion: ......................................... Dauer

ß-Blocker: ..................................... mg

Atropin: ...................................... $\mathrm{mg}$

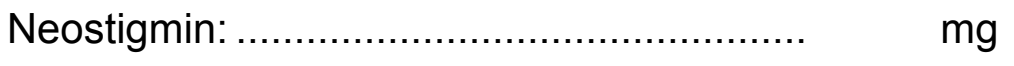

Antiemetika: .................................... $\mathrm{mg}$

Naloxon: ........................................ $\mathrm{mg}$

Flumazenil: ....................................... $\mathrm{mg}$

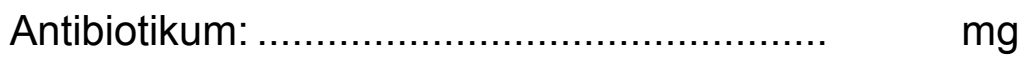

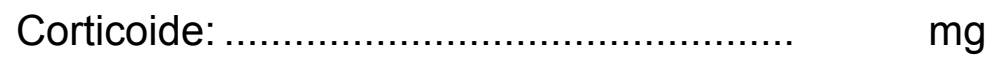

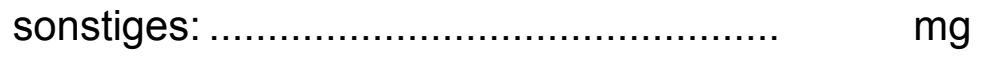

\section{Perioperative Komplikationen (Mehrfachnennung möglich)}

$\begin{array}{rrrr}\text { Hypotension } & \text { Hypertension } & \text { Bradykardie } & \text { Tachykardie } \\ \text { Allergien } & \text { Reanimation } & \text { Thrombose } & \text { Embolie }\end{array}$




\section{Postoperative Medikation (z.B. Aufwachraum)}

\begin{tabular}{|c|c|c|c|c|c|}
\hline Metamizol & $\mathrm{mg}$ & Paracetamol & $\mathrm{mg}$ & Diclofenac & $\mathrm{mg}$ \\
\hline Dipidolor® & $\mathrm{mg}$ & Antiemetika & $\mathrm{mgl}$ & & $\mathrm{mg}$ \\
\hline NTG & $\mathrm{mg}$ & Physostigmin & $\mathrm{mg}$ & Atropin & $\mathrm{mg}$ \\
\hline Droperidol & $\mathrm{mg}$ & iges & & & \\
\hline
\end{tabular}

\section{Fragebogen:}

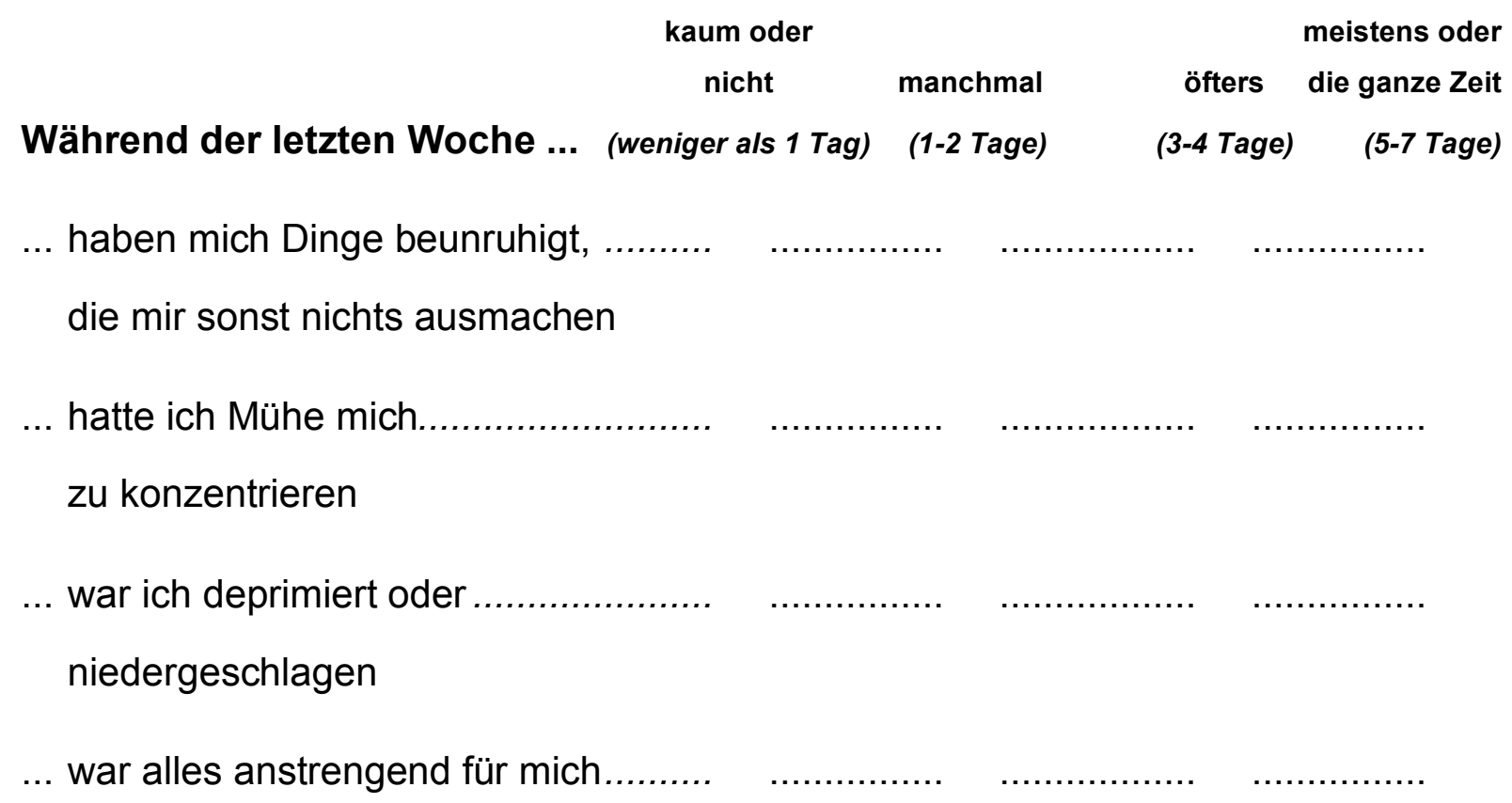


... dachte ich voller Hoffnung

an die Zukunft

... hatte ich oft Angst

... habe ich schlecht geschlafen.

... war ich fröhlich gestimmt

... fühlte ich mich einsam

... bin ich überhaupt nicht

in die Gänge gekommen

Gehen Sie abends gern ins Kino?

ja manchmal nein (wenn "nein", warum nicht?)

Leben Sie allein?

ja nein

Sind Sie oft auf fremde Hilfe angewiesen?

ja nein manchmal

Schauen Sie abends gern Fernsehen?

ja nein manchmal

Reisen Sie gern Reisen mit Auto, Bus oder Flugzeug?

ja manchmal nein (wenn "nein", warum nicht?) 
Wieviele Stunden haben Sie während der letzten vier Wochen durchschnittlich pro Nacht geschlafen? Stunden Schlaf pro Nacht:

Wie würden Sie insgesamt die Qualität Ihres Schlafes während der letzten 4 Wochen beurteilen?

sehr gut ziemlich gut ziemlich schlecht schlecht

Wie oft haben Sie während der letzten vier Wochen unter Schlafstörungen gelitten? (Markieren Sie bitte die auf Sie zutreffenden Antworten und lassen Sie bitte keine Frage aus)

... weil Sie nicht innerhalb einer halben Stunde einschlafen konnten?

3 mal oder mehr pro Woche 1 bis 2-mal pro Woche weniger als einmal pro Woche

in den letzten vier Wochen gar nicht

... weil Sie mitten in der Nacht oder morgens viel zu früh aufgewacht sind.

3 mal oder mehr pro Woche 1 bis 2-mal pro Woche weniger als einmal pro Woche

in den letzten vier Wochen gar nicht

... weil Sie aufstehen mußten, um auf die Toilette zu gehen.

3 mal oder mehr pro Woche 1 bis 2-mal pro Woche weniger als einmal pro Woche

in den letzten vier Wochen gar nicht

... weil Sie Schwierigkeiten beim Atmen hatten 
3 mal oder mehr pro Woche 1 bis 2-mal pro Woche weniger als einmal pro Woche

in den letzten vier Wochen gar nicht

... weil Sie husten mußten oder laut geschnarcht haben

3 mal oder mehr pro Woche 1 bis 2-mal pro Woche weniger als einmal pro Woche

in den letzten vier Wochen gar nicht

... weil Ihnen zu kalt war.

3 mal oder mehr pro Woche 1 bis 2-mal pro Woche weniger als einmal pro Woche

in den letzten vier Wochen gar nicht

... weil Ihnen zu warm war.

3 mal oder mehr pro Woche 1 bis 2-mal pro Woche weniger als einmal pro Woche

in den letzten vier Wochen gar nicht

... weil Sie schlecht geträumt haben

3 mal oder mehr pro Woche 1 bis 2-mal pro Woche weniger als einmal pro Woche

in den letzten vier Wochen gar nicht

... weil Sie Schmerzen haben 
3 mal oder mehr pro Woche 1 bis 2-mal pro Woche weniger als einmal pro Woche

in den letzten vier Wochen gar nicht

.. andere Gründe. Bitte beschreiben

3 mal oder mehr pro Woche 1 bis 2-mal pro Woche weniger als einmal pro Woche

in den letzten vier Wochen gar nicht

Haben Sie Mißempfindungen wie Kribbeln, Ameisenlaufen oder Schmerzen an den Beinen verbunden mit einem Bewegungsdrang

ja nein weiß nicht

Können Sie wegen der komischen Gefühle in den Beinen schlecht einschlafen?

ja nein weiß nicht

Können Sie wegen der komischen Gefühle in den Beinen schlecht durchschlafen?

ja nein weiß nicht

Wann traten diese Symptome das erste Mal auf?:

Wenn ja, wie häufig treten die Symptome auf?:
6-7 Tage pro Woche
4-5 Tage pro Woche
2-3 Tage pro Woche
1 Tag pro Woche
ca. 1-3 mal pro Monat
weniger als $1 \mathrm{mal}$ pro
Monat

Sind diese Symptome abends oder nachts schlimmer als morgens?

ja nein weiß nicht 
Treten diese Symptome nur in Ruhe oder im Bett auf und lassen sie sich durch Bewegung bessern?
ja
nein
weiß nicht

Sind diese Symptome so stark, daß Sie ein wirksames Medikament mit starken Nebenwirkungen einnehmen würden?

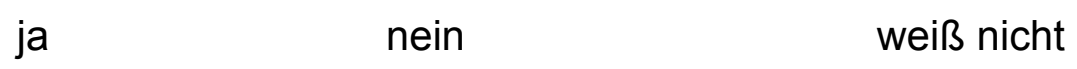

Wenn ja, welches nehmen Sie dann?

Hat noch jemand in der Familie (Eltern/Geschwister/Kinder) ähnliche Beschwerden? ja nein weiß nicht Wenn ja, wer?:

Gibt es ein Medikament, daß Ihre Beschwerden lindert? ja nein weiß nicht Wenn ja, welches?:

Bemerken Sie oder Ihr(e) Bettpartner(in) plötzliche unwillkürliche Zuckungen der Beine?
ja
nein
weiß nicht

Sind Sie am Tage häufig unausgeschlafen und müde?

ja nein weiß nicht

Wie oft hatten Sie während der letzten vier Wochen Schwierigkeiten wachzubleiben, etwa beim Autofahren, beim Essen oder bei gesellschaftlichen Anlässen?
gar nicht bis einmal pro Woche
1 - 2 mal pro Woche
dreimal oder mehr pro Woche 
Anhang

Hatten Sie während der letzten vier Wochen Probleme, mit Elan die alltäglichen Aufgaben zu erledigen?

keine Probleme kaum Probleme zunehmende Probleme große

Probleme 


\section{Danksagung}

Meinem Doktorvater und Betreuer, Herrn Prof. Dr. med. T.A. Crozier, danke ich für die Möglichkeit der Durchführung der vorliegenden Arbeit in der Abteilung für Anästhesiologie, sowie für deren Korrektur und Begutachtung. Ausserdem gilt mein Dank der Zeit seiner geduldigen Betreuung, in der ich mich jederzeit mit Fragen an inn wenden konnte.

Weiterhin danke ich Frau Dr. med. S. Happe, die mir besonders in der Anfangsphase hilfreiche Anregungen zur Durchfürung der Befragung gab und Herrn Dr. med. Dr. rer. nat. J. Schindehütte, die beide die Diagnose bei den Patienten mit einem Restless-Legs-Syndrom bestätigten.

Außerdem möchte ich mich bei Prof. Dr. R. Hilgers aus der Abteilung medizinische Statistik für die Planung der Studie im Hinblick auf die statistischen Parameter und bei Herrn Dr. K. Jung für die spätere Unterstützung bei der statistischen Auswertung der Daten bedanken.

Mein besonderer Dank gilt Prof. Dr. med. U. Braun, der mir die Arbeit vermittelte, mir jederzeit mit Rat und Tat zur Seite stand, mir hilfreiche Anregungen gab und diverse Korrekturlesungen an der Arbeit vorgenommen hat. 


\section{Lebenslauf:}

Mein Name ist Désirée Karimdadian. Ich wurde am 21.08.1978 in Teheran/Iran geboren.

\section{Schulausbildung:}

Von 1985 bis 1989 besuchte ich die Grundschule am Geitelplatz in Wolfenbüttel.

Im Anschluss daran folgte der zweijährige Besuch der Orientierungsstufe Cranachstraße mit dem Wechsel auf das Theodor-Heuss-Gymnasium in Wolfenbüttel 1991.

1998 erlangte ich dort die allgemeine Hochschulreife.

\section{Hochschulstudium:}

1998 begann ich an der Georg-August-Universität in Göttingen das Studium für Englisch und Biologie für höheres Lehramt und wechselte im Sommersemester 1999 zum Studium der Humanmedizin in Göttingen.

Nach Abschluss der Vorklinik absolvierte ich im Frühjahr 2001 das Physikum und nach zwei weiteren Semestern 2002 das 1. Staatsexamen.

Zum Wintersemester 2002 studierte ich für zwei Semester an der Medizinischen Fakultät in Salamanca/Spanien.

2005 folgte das 2.Staatsexamen und im Anschluss das praktische Jahr mit Wahlfach Augenheilkunde an der Universitätsmedizin Göttingen.

2006 beendete ich das Medizinstudium mit dem 3.Staatsexamen.

\section{Berufliche Ausbildung:}

Seit 2006 arbeite ich als Assistenzärztin in der Augenklinik der Universitätsmedizin Göttingen. 